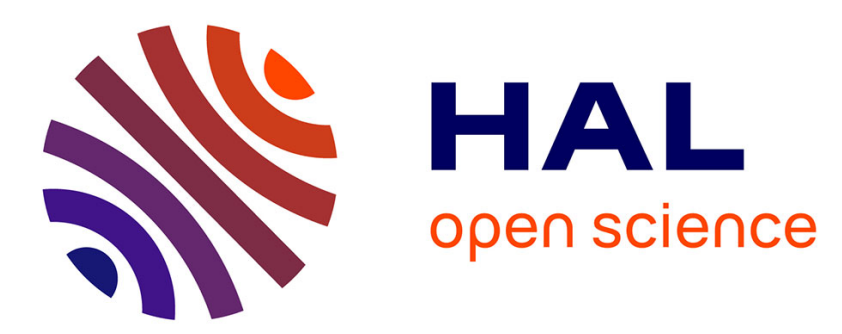

\title{
A framework for the event-triggered stabilization of nonlinear systems
}

Romain Postoyan, Paulo Tabuada, Dragan Nesic, Adolfo Anta

\section{To cite this version:}

Romain Postoyan, Paulo Tabuada, Dragan Nesic, Adolfo Anta. A framework for the event-triggered stabilization of nonlinear systems. IEEE Transactions on Automatic Control, 2015, 60 (4), pp.982-996. 10.1109/TAC.2014.2363603 . hal-01108957

\section{HAL Id: hal-01108957 https://hal.science/hal-01108957}

Submitted on 23 Jan 2015

HAL is a multi-disciplinary open access archive for the deposit and dissemination of scientific research documents, whether they are published or not. The documents may come from teaching and research institutions in France or abroad, or from public or private research centers.
L'archive ouverte pluridisciplinaire HAL, est destinée au dépôt et à la diffusion de documents scientifiques de niveau recherche, publiés ou non, émanant des établissements d'enseignement et de recherche français ou étrangers, des laboratoires publics ou privés. 


\title{
A framework for the event-triggered stabilization of nonlinear systems
}

\author{
Romain Postoyan, Paulo Tabuada, Senior Member, IEEE, Dragan Nešić, Fellow, IEEE, and A. Anta
}

\begin{abstract}
Event-triggered control consists of closing the feedback loop whenever a predefined state-dependent criterion is satisfied. This paradigm is especially well suited for embedded systems and networked control systems since it is able to reduce the amount of communication and computation resources needed for control, compared to the traditional periodic implementation. In this paper, we propose a framework for the event-triggered stabilization of nonlinear systems using hybrid systems tools, that is general enough to encompass most of the existing eventtriggered control techniques, which we revisit and generalize. We also derive two new event-triggering conditions which may further enlarge the inter-event times compared to the available policies in the literature as illustrated by two physical examples. These novel techniques exemplify the relevance of introducing additional variables for the design of the triggering law. The proposed approach as well as the new event-triggering strategies are flexible and we believe that they can be used to address other event-based control problems.
\end{abstract}

Index Terms-Event-triggered control, hybrid systems, sampled-data, networked control systems, nonlinear systems.

\section{INTRODUCTION}

Today's control systems are frequently implemented over networks since these offer many advantages in terms of flexibility and cost. In this setup, the controllers communicate with the sensors and the actuators through the network, not in a continuous fashion but rather at discrete time instants when the channel is available for the control system. Traditionally, the time interval between two successive transmissions is constrained to be less than a fixed constant $T$, which is called the maximum allowable transmission interval (MATI) (see, e.g., [17], [19], [32]). In order to achieve a desired performance, $T$ is generally chosen as small as technology and network load permit. This strategy, although easy to implement, represents a conservative solution that may unnecessarily overload the communication channel. Indeed, one would expect that the transmission instants should not satisfy

R. Postoyan is with the Université de Lorraine, CRAN, UMR 7039 and the CNRS, CRAN, UMR 7039, France romain.postoyan@univ-lorraine.fr. His work was partially supported by the European 7th Framework Network of Excellence "Highlycomplex and networked control systems" (HYCON2) (grant agreement no. 257462)

P. Tabuada is with the Cyber-Physical Systems Laboratory of the Department of Electrical Engineering at the University of California, Los Angeles, CA, USA tabuadadee.ucla.edu. His work was partially supported by the NSF awards 1035916 and 1239085.

D. Nešić is with the Department of Electrical and Electronic Engineering, the University of Melbourne, Parkville, VIC 3010, Australia dnesic@unimelb.edu.au. His work was supported by the Australian Research Council under the Discovery Projects and Future Fellowship schemes.

A. Anta aantamegmail.com a prefixed bound but rather be based on the current state of the system, the channel occupancy and the desired performance. Drawing intuition from this idea, event-triggered control has been developed to reduce the need for communication while guaranteeing satisfactory levels of performance. It involves closing the loop whenever a predefined state-dependent triggering condition is satisfied, e.g., [1], [2], [3], [11], [28], [34]. This technique reduces the usage of the communication bandwidth and of the CPU and provides a high degree of robustness since the state is continuously monitored.

In this paper, we propose a framework for the eventtriggered stabilization of nonlinear systems using hybrid systems tools, and we use it to develop new event-triggering schemes and to revisit and generalize the techniques in [13], [16], [20], [28], [34]. We model event-triggered control nonlinear systems as hybrid systems using the formalism of [8] (see also [5], [6] where linear systems are considered). We show that set stability conveniently describes the desired stability properties of these systems. This property is established using a novel Lyapunov theorem which extends the results in [8] to the case where the Lyapunov function is locally Lipschitz (and not necessarily continuously differentiable), which is essential in event-triggered control where Lyapunov functions are often given by the maximum of continuously differentiable functions (which are locally Lipschitz), as we show. In addition, the Lyapunov theorem relies on a different condition on the flow set which weakens the corresponding one in Theorem 3.18 in [8]. We provide sufficient conditions for guaranteeing the existence of a uniform minimum amount of time between control updates. Although this is not necessary to guarantee stability, this is crucial in practice as the communication hardware cannot generate transmissions which are arbitrarily close in time. We also explain how to redesign the triggering condition to enforce the existence of such a minimum amount of time between two transmissions when the aforementioned conditions are not met because of the behaviour of the solutions near the attractor. Similar results have been derived in different contexts and for specific classes of systems and triggering conditions in [5], [29].

The proposed approach is flexible and offers the possibility to introduce additional variables to construct the triggering condition. We emphasize the potential of these extra variables through two new strategies which may further reduce the usage of the communication resources compared to existing techniques. In the first strategy, we use a threshold variable to close the loop, which allows generating longer transmission intervals than in [28], [34] as illustrated on a jet engine compressor example. This technique is especially well-suited 
for real-time scheduling as we can tune the threshold dynamics according to the available resources. The second strategy is inspired by [19] where a time-triggered implementation is studied. The idea here is to make the dynamics of the clock variable used in [19] state-dependent in order to adapt transmissions to the system state. This strategy is compared with [28] on a van der Pol oscillator. We also show that the approach is general enough to capture many of the eventtriggering laws available in the literature [13], [16], [20], [28], [34]. By doing so, we revisit and generalize these techniques by relaxing the conditions on which they rely and by allowing general holding functions. Indeed, the results of the paper not only apply to zero-order-holds but to various holding functions such as the model-based one proposed in [15] for linear systems. In addition, the proposed new techniques can be used as starting points to address other event-based control problems, such as the stabilization of systems with distributed sensors and actuators ([24]), or the coordination of cyberphysical systems for instance ([21]). Note that a related study has been independently proposed in [26] where strategies similar to those in [6] and [31] are investigated. Contrary to [26], we ensure the existence of a uniform inter-execution time for all our strategies, which is essential in practice as explained above; furthermore we develop different eventtriggering policies and we revisit existing techniques.

The remainder of the paper is organized as follows. Preliminaries are stated in Section II. Event-triggered control systems are modeled as hybrid systems in Section III. The analytical tools used to prove stability as well as the existence of a minimum amount of time between any two transmissions are stated in Section IV. In Section V, we propose new triggering rules and we show how several existing eventtriggering policies can be addressed with the proposed approach. The event-triggering techniques are compared on illustrative examples in Section VI and conclusions are proposed in Section VII. The proofs are given in the Appendix.

\section{PREliminaries}

Let $\mathbb{R}:=(-\infty, \infty), \mathbb{R}_{\geq 0}:=[0, \infty), \mathbb{R}_{>0}:=(0, \infty)$, $\mathbb{Z}_{\geq 0}:=\{0,1,2, \ldots\}, \mathbb{Z}_{>0}:=\{1,2, \ldots\}$. A function $\gamma:$ $\mathbb{R}_{\geq 0} \rightarrow \mathbb{R}_{\geq 0}$ is of class $\mathcal{K}$ if it is continuous, zero at zero and strictly increasing, and it is of class $\mathcal{K}_{\infty}$ if in addition it is unbounded. The notation $\mathbb{I}$ denotes the identity mapping from $\mathbb{R}_{\geq 0}$ to $\mathbb{R}_{\geq 0}$. Let $(x, y) \in \mathbb{R}^{n+m},(x, y)$ stand for $\left[x^{\mathrm{T}}, y^{\mathrm{T}}\right]^{\mathrm{T}}$. The distance of a vector $x$ to a set $\mathcal{A} \subset \mathbb{R}^{n}$ is denoted by $|x|_{\mathcal{A}}:=\inf \{|x-y|: y \in \mathcal{A}\}$.

The notation $f^{-1}(\mathcal{M})$ for a function $f: \mathbb{R}^{n} \rightarrow \mathbb{R}^{m}$ and a set $\mathcal{M} \subset \mathbb{R}^{m}$ stands for $\{x: f(x) \in \mathcal{M}\}$. When $f: \mathbb{R}^{n} \rightarrow \mathbb{R}, f_{\leq}^{-1}(m):=\{x: f(x) \leq m\}$ and $f_{\geq}^{-1}(m):=\{x: \bar{f}(x) \geq m\}$ for $m \in \mathbb{R}$. The notation $\left.\right|^{\cdot} \mid$ stands for the Euclidean norm. We use $U^{\circ}(x ; v)$ to denote the generalized directional derivative of Clarke of a locally Lipschitz function $U$ at $x$ in the direction $v$, i.e. $U^{\circ}(x ; v):=\lim \sup _{h \rightarrow 0^{+}, y \rightarrow x}(U(y+h v)-U(y)) / h$, which reduces to the standard directional derivative $\langle\nabla U(x), v\rangle$ when $U$ is continuously differentiable; see [4] for more detail. We will use the following result which corresponds to Proposition 1.1 in [18].

Lemma 1: Consider two continuously differentiable functions $U_{1}: \mathbb{R}^{n} \rightarrow \mathbb{R}$ and $U_{2}: \mathbb{R}^{n} \rightarrow \mathbb{R}$. Let $A:=$ $\left\{x: U_{1}(x)>U_{2}(x)\right\}, B:=\left\{x: U_{1}(x)<U_{2}(x)\right\}$ and $\Gamma:=\left\{x: U_{1}(x)=U_{2}(x)\right\}$. For any $v \in \mathbb{R}^{n}$, the function $U:$ $x \mapsto \max \left\{U_{1}(x), U_{2}(x)\right\}$ satisfies $U^{\circ}(x ; v)=\left\langle\nabla U_{1}(x), v\right\rangle$ for all $x \in A, U^{\circ}(x ; v)=\left\langle\nabla U_{2}(x), v\right\rangle$ for all $x \in B$, and $U^{\circ}(x ; v)=\max \left\{\left\langle\nabla U_{1}(x), v\right\rangle,\left\langle\nabla U_{2}(x), v\right\rangle\right\}$ for all $x \in \Gamma$.

The lemma below will be useful for lower bounding Lyapunov functions. Its proof follows from the fact that $\alpha\left(s_{1}+s_{2}\right) \leq \alpha\left(2 s_{1}\right)+\alpha\left(2 s_{2}\right)$ for any $\alpha \in \mathcal{K}_{\infty}, s_{1}, s_{2} \geq 0$.

Lemma 2: For any $\alpha_{1}, \alpha_{2} \in \mathcal{K}_{\infty}, \alpha_{1}\left(s_{1}\right)+\alpha_{2}\left(s_{2}\right) \geq$ $\underline{\alpha}\left(s_{1}+s_{2}\right)$ for any $s_{1}, s_{2} \geq 0$ where $\underline{\alpha}: s \mapsto$ $\min \left\{\alpha_{1}(s / 2), \alpha_{2}(s / 2)\right\} \in \mathcal{K}_{\infty}$.

The following technical lemma is an extension of Lemma 4.3 in [12], which is convenient when designing Lyapunov functions to investigate set stability.

Lemma 3: Let $\mathcal{O} \subseteq \mathbb{R}^{n}$ and $\mathcal{A} \subset \mathcal{O}$ be non-empty and compact sets and let $\bar{V}: \mathcal{O} \rightarrow \mathbb{R}_{\geq 0}$ be a continuous function which is equal to zero only on $\mathcal{A}$. There exist $\alpha_{1}, \alpha_{2} \in \mathcal{K}_{\infty}$ such that

$$
\alpha_{1}\left(|x|_{\mathcal{A}}\right) \leq V(x) \leq \alpha_{2}\left(|x|_{\mathcal{A}}\right) \quad \forall x \in \mathcal{O} .
$$

When $\mathcal{O}$ is non-empty, closed and unbounded and $V$ tends to infinity as $|x|_{\mathcal{A}} \rightarrow \infty$, (1) holds.

We recall the definition of the tangent cone to a set at a given point and state a useful lemma.

Definition 1: [8] The tangent cone to a set $S \subset \mathbb{R}^{n}$ at a point $x \in \mathbb{R}^{n}$, denoted $T_{S}(x)$, is the set of all vectors $w \in \mathbb{R}^{n}$ for which there exist $x_{i} \in S, \tau_{i}>0$ with $x_{i} \rightarrow x, \tau_{i} \rightarrow 0$ as $i \rightarrow \infty$ such that $w=\lim _{i \rightarrow \infty}\left(x_{i}-x\right) / \tau_{i}$.

Lemma 4: Let $f: \mathbb{R}^{n} \rightarrow \mathbb{R}^{n}$ be continuous, $C=\{q$ : $V(x) \geq W(x)\}$ and $D=\{q: V(x) \leq W(x)\}$ for some continuously differentiable functions $V$ and $W$ from $\mathbb{R}^{n}$ to $\mathbb{R}$. For any $x \in C \cap D, f(x) \in T_{C}(x)$ implies that $\langle\nabla V(x), f(x)\rangle \geq\langle\nabla W(x), f(x)\rangle$.

\section{SYSTEM MODELS}

We will write event-triggered control systems as hybrid systems using the formalism of [8], like in [5], [6], [26]. Thus, we consider systems of the form

$$
\dot{q}=F(q) \text { for } q \in C, \quad q^{+}=G(q) \text { for } q \in D,
$$

where $q \in \mathbb{R}^{n_{q}}$ is the state and $n_{q} \in \mathbb{Z}_{>0}$. The sets $C \subset \mathbb{R}^{n_{q}}$ and $D \subset \mathbb{R}^{n_{q}}$ are closed and respectively denote the flow and the jump sets. The vector fields $F$ and $G$ are assumed to be continuous on $C$ and $D$, respectively. The continuity of $F$ and $G$ together with the closedness of the sets $C$ and $D$ ensure that the system is 'well-posed', see Chapter 6 in [8].

In event-triggered control, the transmissions occur whenever a state-dependent criterion is satisfied. The hybrid model (2) is well-equipped to describe these systems. Indeed, a transmission can be modeled as a jump of (2) that occurs whenever the event-triggering condition is violated, which corresponds to the fact that the state of system (2) enters in 
the jump set $D$. When $q \in C$, the system flows and, when $q \in C \cap D$, the system can either jump or flow, the latter only if flowing keeps $q$ in $C$.

In this paper, the model (2) is obtained by following an emulation-like approach (see, e.g., [17], [19], [28], [32]), i.e. a controller is first assumed to be designed without considering the resource limitations, afterwards, we take into account the effects induced by the latter and we derive an appropriate event-triggering condition to ensure stability for the obtained system.

Suppose that there is no resource constraints and consider the plant

$$
\dot{x}_{p}=f_{p}\left(x_{p}, u\right) \text {, }
$$

where $x_{p} \in \mathbb{R}^{n_{p}}$ is the plant state and $u \in \mathbb{R}^{n_{u}}$ is the control input, for which a stabilizing dynamic state-feedback controller is designed

$$
\dot{x}_{c}=f_{c}\left(x_{c}, x_{p}\right), \quad u=g_{c}\left(x_{c}, x_{p}\right),
$$

where $x_{c} \in \mathbb{R}^{n_{c}}$ is the controller state. Note that when the controller is static, (4) reduces to $u=g_{c}\left(x_{p}\right)$. We now take into account the digital nature of the communication medium. At each transmission instant ${ }^{1}$, the controller receives the plant measurements, updates its knowledge of it, sends the control input and the actuators update the signal sent to the plant. As a consequence, the plant and the controller no longer have access to $u$ and $x_{p}$, respectively. Instead, they only know the corresponding sampled versions, i.e. $\hat{u}$ and $\hat{x}_{p}$, which are reset to the actual values of $u$ and $x_{p}$ at each transmission instant, i.e.

$$
\hat{x}_{p}^{+}=x_{p}, \quad \hat{u}^{+}=u .
$$

Between two transmissions, $\hat{u}$ and $\hat{x}_{p}$ are generated by given holding functions which are respectively implemented at the actuators and at the controller

$$
\dot{\hat{x}}_{p}=\hat{f}_{p}\left(x_{p}, x_{c}, \hat{x}_{p}, \hat{u}\right), \quad \dot{\hat{u}}=\hat{f}_{u}\left(x_{p}, x_{c}, \hat{x}_{p}, \hat{u}\right) .
$$

The use of zero-order-hold devices lead to $\hat{f}_{p}=0$ and $\hat{f}_{u}=$ 0 . This formulation also allows to consider the model-based technique in [15], as we show in Section V-C. We allow $\hat{f}_{p}$ and $\hat{f}_{u}$ to depend on $x_{p}, x_{c}, \hat{x}_{p}, \hat{u}$ for the sake of generality to capture the cases where they depend on a part of these vector variables. We introduce the sampling-induced error to model the impact of the sampling

$$
e:=\left(\begin{array}{c}
\hat{x}_{p}-x_{p} \\
\hat{u}-u
\end{array}\right)
$$

which is reset to 0 at each jump in view of (5). We assume that the dynamics of the controller (4) is sufficiently fast compared to the transmissions rate so that we can ignore its sampling due to its implementation on a digital platform (as in [19], [32] for instance). To define the event-triggering law, we may introduce auxiliary variables which we denote by a single vector variable $\eta \in \mathbb{R}^{n_{\eta}}$. Indeed, it is common in the hybrid literature to use additional variables like clocks to ensure or analyze the

\footnotetext{
${ }^{1}$ We suppose that this process occurs in a synchronized manner and we ignore the effects of the induced delays, noting that they may be analyzed by following similar lines as in [28].
}

stability, see [8]. We will see for instance in Section V-E that we need to introduce a threshold variable to revisit the work in [34]. The $\eta$-system has for dynamics $\dot{\eta}=h(x, e, \eta)$ between two successive transmission instants and $\eta^{+}=\ell(x, e, \eta)$ at each transmission instant where $h$ and $\ell$ are designed by the user.

In that way, we obtain system (2) with

$$
q=\left(\begin{array}{c}
x \\
e \\
\eta
\end{array}\right) F(q)=\left(\begin{array}{c}
f(x, e) \\
g(x, e) \\
h(x, e, \eta)
\end{array}\right) G(q)=\left(\begin{array}{c}
x \\
0 \\
\ell(x, e, \eta)
\end{array}\right)
$$

where $x=\left(x_{p}, x_{c}\right), F$ and $G$ are assumed to be continuous and $f(x, e)=\left(f_{p}\left(x_{p}, g_{c}\left(x_{c}, x_{p}+e_{x_{p}}\right)+e_{u}\right), f_{c}\left(x_{c}, x_{p}+e_{x_{p}}\right)\right)$, $g(x, e)=\left(\hat{f}_{p}\left(x_{p}, x_{c}, x_{p}+e_{x_{p}}, g_{c}\left(x_{c}, x_{p}+e_{x_{p}}\right)+e_{u}\right)-\right.$ $f_{p}\left(x_{p}, g_{c}\left(x_{c}, x_{p}+e_{x_{p}}\right)+e_{u}\right), \hat{f}_{u}\left(x_{p}, x_{c}, x_{p}+e_{x_{p}}, g_{c}\left(x_{c}, x_{p}+\right.\right.$ $\left.\left.\left.e_{x_{p}}\right)+e_{u}\right)-\frac{\partial g_{c}}{\partial x_{c}}\left(x_{c}, x_{p}+e_{x_{p}}\right) f_{c}\left(x_{c}, x_{p}+e_{x_{p}}\right)\right)$ with $e_{x_{p}}=$ $\hat{x}_{p}-x_{p}$ and $e_{u}=\hat{u}-u$.

The main problem in event-triggered control is to define the triggering condition, i.e. the flow and jump sets $C$ and $D$ in (2), (and the dynamics of $\eta$ if needed) in order to reduce the resource usage, i.e. the amount of jumps, while ensuring asymptotic stability properties together with the existence of a uniform minimum inter-execution time.

Remark 1: The assumptions allow for triggering rules that depend both on $x$ and $e$. However, the specific choice of triggering rule needs to be done according to the implementation scenario. In the case of dynamic controllers, a triggering rule depending on $x_{c}$ requires continuous communication between the sensors and the controller. This is difficult to achieve in practice since sensors do not have, in general, access to the state of the controller. We have chosen to present the problem in a general setting because it allows to recover as particular cases the stabilization using a static controller (as in [13], [16], [20], [28], [34] for example) and the cases where only the plant states (i.e. $e=\hat{x}_{p}-x_{p}$ ) or the inputs (i.e. $e=\hat{u}-u$ ) are sampled.

\section{AnAlytical TOOLS}

By writing general event-triggered control systems as hybrid systems using the formalism of [8], we can use a wide range of tools to study system (2). In this paper, we focus on set stability, which is a natural property when investigating eventtriggered control systems as we show in the forthcoming section. We present a new Lyapunov theorem to guarantee set stability, which extends the corresponding statements in [8]. This result is used in Section V to analyse the stability of various event-triggered control systems. We conclude this section with sufficient conditions for the existence of a uniform amount of time between any two transmissions.

\section{A. Definitions}

We recall some definitions related to the hybrid framework of [8]. A subset $E \subset \mathbb{R}_{\geq 0} \times \mathbb{Z}_{\geq 0}$ is a hybrid time domain if for all $(T, J) \in E, E \cap([0, T] \times\{0, \ldots, J\})=$

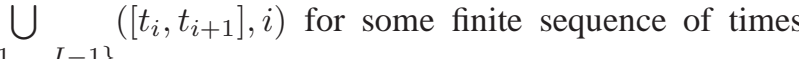
$i \in\{0,1, \ldots, I-1\}$ 
$0=t_{0} \leq t_{1} \leq \ldots \leq t_{I}$. A function $\phi: E \rightarrow \mathbb{R}^{n_{q}}$ is a hybrid arc if $E$ is a hybrid time domain and if for each $j \in \mathbb{Z}_{\geq 0}, t \mapsto \phi(t, j)$ is locally absolutely continuous on $I^{j}:=\{t:(t, j) \in E\}$. The hybrid $\operatorname{arc} \phi: \operatorname{dom} \phi \rightarrow \mathbb{R}^{n_{q}}$ is a solution to (2) if: (i) $\phi(0,0) \in C \cup D$; (ii) for any $j \in \mathbb{Z}_{\geq 0}$, $\phi(t, j) \in C$ and $\frac{d}{d t} \phi(t, j)=F(\phi(t, j))$ for almost all $t \in \bar{I}^{j}$; (iii) for every $(t, j) \in \operatorname{dom} \phi$ such that $(t, j+1) \in \operatorname{dom} \phi$, $\phi(t, j) \in D$ and $\phi(t, j+1)=G(\phi(t, j))$. A solution $\phi$ is maximal when it cannot be extended, and is complete when dom $\phi$ is unbounded. We recall the following invariance definition for hybrid systems, see Definition 6.25 in [8].

Definition 2: A set $S \subset \mathbb{R}^{n_{q}}$ is strongly forward preinvariant if for every solution $\phi$ to $(2), \phi(t, j) \in S$ for some $(t, j) \in \operatorname{dom} \phi$ implies that $\phi\left(t^{\prime}, j^{\prime}\right) \in S$ for any $\left(t^{\prime}, j^{\prime}\right) \in \operatorname{dom} \phi$ with $t+j \leq t^{\prime}+j^{\prime}$.

We introduce the definition below to characterize hybrid systems that generate solutions which have a uniform semiglobal minimum amount of time between two jumps, except, possibly, on a given set of the state space (the attractor in this study).

Definition 3: The solutions to (2) have a uniform semiglobal dwell-time outside $\mathcal{A}$ where $\mathcal{A} \subseteq \mathbb{R}^{n_{q}}$ is strongly forward pre-invariant for system (2), if for any $\Delta \geq 0$, there exists $\tau(\Delta)>0$ such that for any solution $\phi$ to (2) with $|\phi(0,0)|_{\mathcal{A}} \leq \Delta$ and any $(s, i),(t, j) \in \operatorname{dom} \phi$ with $s+i \leq t+j$,

$$
\phi(t, j) \notin \mathcal{A} \Rightarrow j-i \leq(t-s) / \tau(\Delta)+1 .
$$

The solutions to (2) have a uniform semiglobal dwell-time if for any $\Delta \geq 0$, there exists $\tau(\Delta)>0$ such that for any solution $\phi$ to (2) with $|\phi(0,0)| \leq \Delta$ and for any $(s, i),(t, j) \in$ $\operatorname{dom} \phi$ with $s+i \leq t+j, j-i \leq(t-s) / \tau(\Delta)+1$.

We will see in the following sections that it is natural to work with set stability when studying event-triggered control systems. We consider the definition below which comes from Definition 3.6 in [8].

Definition 4: The closed set $\mathcal{A} \subset \mathbb{R}^{n_{q}}$ is uniformly globally pre-asymptotically stable (UGpAS) for system (2) if the following holds.

(i) [Uniform global stability] There exists $\alpha \in \mathcal{K}_{\infty}$ such that for any solution $\phi$ to $(2),|\phi(t, j)|_{\mathcal{A}} \leq \alpha\left(|\phi(0,0)|_{\mathcal{A}}\right)$ for all $(t, j) \in \operatorname{dom} \phi$.

(ii) [Uniform global pre-attractivity] For each $\varepsilon, r>0$, there exists $T>0$ such that for any solution $\phi$ to (2) with $|\phi(0,0)|_{\mathcal{A}} \leq r,(t, j) \in \operatorname{dom} \phi$ and $t+j \geq T$ imply $|\phi(t, j)|_{\mathcal{A}} \leq \varepsilon$.

We say that $\mathcal{A}$ is uniformly globally asymptotically stable (UGAS) when, in addition, the maximal solutions to (2) are complete.

\section{B. Lyapunov conditions}

As mentioned above, we resort to a locally Lipschitz Lyapunov function $R$ to prove that a given closed set $\mathcal{A}$ is $\mathrm{UG}(\mathrm{p}) \mathrm{AS}$ for system (2). This function $R$ is typically composed of a known Lyapunov function for the closed-loop system when ignoring the sampling and extra terms which involve the variables induced by the sampling, i.e. the error $e$ and the potential additional variable $\eta$. Explicit constructions of $R$ are presented in Section V. We use the following result to conclude stability of $\mathcal{A}$ which states that $R$ is required: (i) to be positive definite and radially unbounded with respect to $\mathcal{A}$; (ii) to decrease on flows as long as $F(q)$ belongs to the tangent cone of $C$ at $q \in C$ (denoted $T_{C}(q)$ ) and as $q \notin \mathcal{A}$; (iii) not to increase at jumps. The item (ii) is motivated by the fact that, when $F(q)$ does not belong to $T_{C}(q)$ (which can only happen at the boundary of $C$ ), a solution which would reach this point cannot keep flowing as it would leave the set $C$ and a jump will immediately occur or the solution will stop to exist. Hence, it is not necessary to require $R$ to decrease at such points. The stability of $\mathcal{A}$ is then deduced by assuming that there exists a minimum amount of time between two jumps. This last condition is sufficient but not necessary to obtain the desired stability result, nevertheless it is fundamental when dealing with event-triggered control as the hardware always has a certain latency which prevents the occurrence of arbitrarily close in time transmissions. The theorem below formalizes these ideas.

Theorem 1: Consider system (2) and let $\mathcal{A}$ be a nonempty closed subset of $\mathbb{R}^{n_{q}}$. Suppose that there exists $R$ : $C \cup D \cup G(D) \rightarrow \mathbb{R}_{>0}$ which is locally Lipschitz on an open set containing $C$, such that the following conditions hold.

(i) There exist $\underline{\alpha}_{R}, \bar{\alpha}_{R} \in \mathcal{K}_{\infty}$ such that for any $q \in C \cup$ $D \cup G(D)$

$$
\underline{\alpha}_{R}\left(|q|_{\mathcal{A}}\right) \leq R(q) \leq \bar{\alpha}_{R}\left(|q|_{\mathcal{A}}\right) .
$$

(ii) There exists a continuous positive definite function $\alpha_{R}$ : $\mathbb{R}_{\geq 0} \rightarrow \mathbb{R}_{\geq 0}$ such that

$$
\begin{aligned}
R^{\circ}(q ; F(q)) \leq & -\alpha_{R}(R(q)) \\
& \text { for all } q \in C \text { such that } F(q) \in T_{C}(q) .
\end{aligned}
$$

(iii) For all $q \in D$

$$
R(G(q)) \leq R(q)
$$

(iv) Solutions to (2) have a uniform semiglobal dwell-time outside $\mathcal{A}$.

Then the set $\mathcal{A}$ is UGpAS. When, in addition $\mathcal{A}$ is compact, $G(D) \subset C \cup D$ and $F(q) \in T_{C}(q)$ for any $q \in C \backslash D$, the set $\mathcal{A}$ is UGAS.

Compared to Proposition 3.27 in [7], the Lyapunov function is not required to be continuously differentiable on an open set containing $C$ but only locally Lipschitz on this set. We will see in Section $\mathrm{V}$ that the analysis of event-triggered control systems often leads to such Lyapunov functions. Moreover, $R$ is not required to strictly decrease on the whole set $C \backslash \mathcal{A}$ (see item (ii) of Theorem 1), but only on a subset of $C \backslash \mathcal{A}$ where $F(q)$ belongs to $T_{C}(q)$. This condition appears to be crucial in the analyses carried out in Section V. Finally, no condition is imposed on the persistency of flows in the attractor $\mathcal{A}$.

In all the strategies investigated in this paper (except the one in Section V-E), the attractor is compact. In this case, the facts that $\mathcal{A}$ is UG(p)AS and that system (2) is well-posed (see Chapter 6 in [8]) ensure that this stability property is robust to small uncertainties on the measurements, the plant dynamics, and the jump instants, see for more detail Theorem 7.21 in [8]. 
It has to be noted that the only region of the state space where the existence of a uniform semiglobal dwell-time may not be guaranteed is in the attractor $\mathcal{A}$. While it may be difficult to ensure that all solutions to (2) which lie in $\mathcal{A}$ have a uniform semiglobal dwell-time, $\mathcal{A}$ is typically a subset of $C$ as it is the case for all the event-triggering schemes studied in this paper (except in Section V-D where the solutions to the corresponding hybrid system have a uniform semiglobal dwelltime on the whole state space). In that way, for a given initial condition in $\mathcal{A}$, there always exists a solution starting from this point which never jumps. This means that in practice, if a solution to system (2) reaches $\mathcal{A}$, we can turn off the triggering mechanism. Further insight about the existence of dwell-time outside the set $\mathcal{A}$ is given in the following subsection.

\section{Existence of uniform semiglobal dwell-times}

The following proposition lists a set of requirements for the existence of uniform semiglobal dwell times outside the attractor $\mathcal{A}$. These conditions are used in Section $\mathrm{V}$ to verify the existence of such times.

Proposition 1: Consider system (2) and suppose the following holds.

(i) Items (i)-(iii) of Theorem 1 hold.

(ii) For any $\Delta \geq 0$ there exists $\psi: \mathcal{S}(\Delta) \rightarrow \mathbb{R}_{\geq 0}$, where $\mathcal{S}(\Delta)$ is an open set containing $R_{<}^{-1}(\Delta) \backslash \mathcal{A}$, which is locally Lipschitz and verifies the following:

(ii-a) there exists $a \geq 0$ such that $\psi(G(q)) \leq a$ for any $q \in \mathcal{S}(\Delta) \cap D$ with $G(q) \in \mathcal{S}(\Delta) ;$

(ii-b) there exists $b>a$ such that for any $q \in \mathcal{S}(\Delta)$, $\psi(q)<b$ implies $q \in C \backslash D ;$

(ii-c) there exists a continuous non-decreasing function $\lambda: \mathbb{R}_{>0} \rightarrow \mathbb{R}_{>0}$ such that for almost all $q \in \mathcal{S}(\Delta)$, $\langle\nabla \psi(q), F(q)\rangle \leq \lambda(\psi(q))$.

Then the solutions to (2) have a uniform semiglobal dwelltime outside $\mathcal{A}$. In addition, when item (i) holds with $\mathcal{A}$ compact and item (ii) is verified with $\mathcal{S}(\Delta)$ an open set containing $R_{<}^{-1}(\Delta)$, the solutions to (2) have a uniform semiglobal dwell-time.

The conditions of Proposition 1 can be interpreted as follows. Item (i) simply states that all the conditions of Theorem 1 are verified except item (iv) which is established using Proposition 1. The function $\psi$ in item (ii) of Proposition 1 is used to guarantee that there exists a uniform minimum amount of time between two successive jumps outside $\mathcal{A}$. By estimating the time it takes for $\psi$ to grow from $a$ to $b$ using the growth condition in item (ii-c), we are able to obtain the desired result. Note that $\psi$ does not need to be defined on $\mathcal{A}$ since the purpose of Proposition 1 is to ensure the existence of uniform semiglobal dwell-time outside $\mathcal{A}$.

For some systems, it may happen that the conditions of Proposition 1 are not met because of the behaviour of the solutions to (2) near the set $\mathcal{A}$ (as discussed later in Remark 6 for example). In this case, we can modify the triggering condition (i.e. the sets $C$ and $D$ ) to guarantee a weaker asymptotic stability property for system (2) than the one ensured by Theorem 1. Suppose that we can prove that the solutions to (2) have a uniform semiglobal dwell-time outside $\widehat{\mathcal{A}}$ where $\widehat{\mathcal{A}}:=R_{\leq}^{-1}(\varepsilon)$ for some $\varepsilon>0$. We modify the flow and the jumps sets for system (2) as follows

$$
\widehat{C}=C \cup R_{\leq}^{-1}(\varepsilon), \quad \widehat{D}=D \cap R_{\geq}^{-1}(\varepsilon) .
$$

In that way, we obtain the result below.

Proposition 2: Consider system (2) with the flow and jump sets defined as in (13) with $\varepsilon>0$ and suppose the following holds.

(i) Items (i)-(iii) of Theorem 1 hold with $R$ continuously differentiable, and $\mathcal{A}$ compact.

(ii) Solutions to (2) have a uniform semiglobal dwell-time outside $\widehat{\mathcal{A}}$.

Then the set $\widehat{\mathcal{A}}$ is UGpAS. When, in addition $G(D) \subset C \cup D$ and $F(q) \in T_{\widehat{C}}(q)$ for any $q \in \widehat{C} \backslash \widehat{D}$, the set $\widehat{\mathcal{A}}$ is UGAS. Furthermore, in this case, when item (ii) of Theorem 1 holds for all $q \in \widehat{C}$ with $F(q) \in T_{\widehat{C}}(q)$, the maximal solutions converge in finite hybrid time to $\widehat{\mathcal{A}}$, i.e. for any maximal solution $\phi$ there exists $\left(t^{\star}, j^{\star}\right) \in \operatorname{dom} \phi$ such that for all $(t, j) \in \operatorname{dom} \phi$ with $t^{\star}+j^{\star} \leq t+j, \phi(t, j) \in \widehat{\mathcal{A}}$.

Noting that Theorem 1 ensures the UG(p)AS of $\mathcal{A}=R^{-1}(0)$, we see that Proposition 2 guarantees the same stability property for the larger set $R_{<}^{-1}(\varepsilon)$ provided that we inflate the flow set and restrict the jump set as in (13). We emphasize that Proposition 2 still applies when $R$ is the maximum of a finite number of continuously differentiable functions (as it is the case in Sections V-A, V-C, V-D for instance). Proposition 2 can be used to relax the conditions which are imposed in Sections $\mathrm{V}$ to ensure that solutions have a uniform semiglobal dwelltime outside the attractor $\mathcal{A}$. We will only explicitly mention this relaxation for the event-triggering scheme in Section $\mathrm{V}-\mathrm{C}$; similar conclusions can be drawn for the other techniques for which $\mathcal{A}$ is compact.

\section{MAin RESUlts}

In this section, we first present two new triggering rules. We then revisit and generalize the strategies in [13], [16], [20], [28], [34].

\section{A. Using a threshold variable}

We assume that the system $\dot{x}=f(x, e)$ (see Section III) is input-to-state stable (ISS) with respect to the samplinginduced errors, like in [28]. This is equivalent to the following assumption (see Theorem 1 in [27]).

Assumption 1: There exist a continuously differentiable Lyapunov function $V: \mathbb{R}^{n_{x}} \rightarrow \mathbb{R}$ and $\underline{\alpha}_{V}, \bar{\alpha}_{V}, \alpha, \gamma \in \mathcal{K}_{\infty}$ such that for all $x \in \mathbb{R}^{n_{x}}$

$$
\underline{\alpha}_{V}(|x|) \leq V(x) \leq \bar{\alpha}_{V}(|x|),
$$

and for all $(x, e) \in \mathbb{R}^{n_{x}+n_{e}}$

$$
\langle\nabla V(x), f(x, e)\rangle \leq-\alpha(V(x))+\gamma(|e|) .
$$

From (15), we deduce that $\sigma \circ \alpha(V(x)) \geq \gamma(|e|)$ with $\sigma \in \mathcal{K}_{\infty}$ and $\sigma(s)<s$ for $s>0$, implies

$$
\langle\nabla V(x), f(x, e)\rangle \leq-(\mathbb{I}-\sigma) \circ \alpha(V(x)) .
$$


We rewrite the condition $\sigma \circ \alpha(V(x)) \geq \gamma(|e|)$ as

$$
V(x) \geq \alpha^{-1} \circ \sigma^{-1} \circ \gamma(|e|)=: W(e) .
$$

In [28], transmissions occur when $W(e) \geq V(x)$ (see Section $\mathrm{V}-\mathrm{C})$. Here, the triggering condition is derived by defining a threshold on the Lyapunov function $V(x)$ and $W(e)$. The idea is similar to what is done in [34], as we will see in Section V-E, where a threshold is applied to $V(x)$ only. Here, we tolerate $V(x)$ to become larger than the threshold (which is not the case in [34]) which may have the effect of enlarging the interexecution time as illustrated in Section VI-A. We define the threshold variable $\eta \in \mathbb{R}_{\geq 0}$ as the solution of the following differential equation on flows

$$
\dot{\eta}=-\delta(\eta),
$$

where $\delta$ is any locally Lipschitz class- $\mathcal{K}$ function, and at jumps,

$$
\eta^{+}=W(e) .
$$

A natural choice of triggering rule is $W(e) \geq \eta$. Nevertheless, in the case where $W(e) \leq V(x), V$ decreases according to (16) and therefore we do not need to close the loop. This suggests considering the following triggering condition instead

$$
W(e) \geq \max \{\eta, V(x)\} .
$$

The problem is modeled as follows

$$
\left.\left.\begin{array}{l}
\dot{x}=f(x, e) \\
\dot{e}=g(x, e) \\
\dot{\eta}=-\delta(\eta)
\end{array}\right\} q \in C, \quad \begin{array}{l}
x^{+}=x \\
e^{+}=0 \\
\eta^{+}=W(e)
\end{array}\right\} q \in D,
$$

where $q=(x, e, \eta)$, and

$$
\begin{aligned}
& C=\{q: \max \{V(x), \eta\} \geq W(e), \eta \geq 0\} \\
& D=\{q: \max \{V(x), \eta\} \leq W(e), \eta \geq 0\} .
\end{aligned}
$$

To guarantee the existence of a uniform semiglobal minimum interval of time between two transmissions outside the origin, the following conditions are assumed to hold like in [28].

Assumption 2: For any compact set $S \subset \mathbb{R}^{n_{x}+n_{e}}$, there exist $L_{1}, L_{2}, L_{3} \in \mathbb{R}_{\geq 0}$ such that for all $(x, e) \in S$

$$
\begin{aligned}
& |f(x, e)| \leq L_{1}(|x|+|e|), \quad \underline{\alpha}_{V}^{-1} \circ W(e) \leq L_{2}|e|, \\
& |g(x, e)| \leq L_{3}(|x|+|e|) .
\end{aligned}
$$

The following theorem ensures the stability of system (21). Its proof is an application of Theorem 1 with $R(q)=$ $\max \{V(x), W(e), \eta\}$.

Theorem 2: Consider system (21), (22), let $\mathcal{A}=\{q$ : $(x, e, \eta)=0\}$, and suppose Assumptions 1-2 hold with $W$ continuously differentiable. The solutions to (21) have a uniform semiglobal dwell-time outside $\mathcal{A}$ and $\mathcal{A}$ is UGAS. $\square$

Remark 2: In Theorem 2, $W$ is required to be continuously differentiable. When it is not the case, we can always upper-bound it by a class- $\mathcal{K}_{\infty}$ function which is continuously differentiable on $\mathbb{R}_{>0}$ (see Lemma B.1.2. in [9]) and define the triggering condition using this upper-bound instead of $W(e)$. When the differentiability of $W$ (or its upper-bound) is not guaranteed at the origin, the triggering condition can be modified as in (13) to ensure a uniform practical asymptotic stability property for the origin of the obtained system in view of Proposition 2.

Remark 3: In [26], an event-triggering technique is proposed which combines the ideas of [28] and [31]. It essentially consists in allowing $V$ to grow as long as it remains below its value at the last jump multiplies by a constant $\lambda \in(0,1)$, which leads to a piecewise constant threshold as opposed to the continuously decreasing threshold presented above.

\section{B. Using a clock variable}

In [19], sampled-data systems with time-triggered execution are modeled as a hybrid system similar to (2) by introducing a clock variable $\tau$. The flow and the jump sets are defined as $\tau$ being smaller or not than a given fixed bound $T$ known as the MATI. This constant $T$ corresponds to the time it takes for the solution of the ordinary differential equation $\dot{\zeta}=-2 L \zeta-\gamma\left(\zeta^{2}+1\right)$ to decrease from $\rho^{-1}$ to $\rho$, where $L$ and $\gamma$ are some constants (see (5) in [19]) and $\rho \in(0,1)$ is arbitrarily small. In this subsection, we modify the strategy in [19] by making the ordinary differential equation that defines $\zeta$ state-dependent. This allows us to consider a larger class of systems and to potentially enlarge the inter-execution intervals compared to [19] as the clock velocity depends on the current state of the system (see Remark 4 below). We make the following assumption.

Assumption 3: There exist locally Lipschitz functions $V$ : $\mathbb{R}^{n_{x}} \rightarrow \mathbb{R}, W: \mathbb{R}^{n_{e}} \rightarrow \mathbb{R}$ and continuous functions $H:$ $\mathbb{R}^{n_{x}} \rightarrow \mathbb{R}_{\geq 0}, L, G: \mathbb{R}^{n_{x}+n_{e}} \rightarrow \mathbb{R}_{\geq 0}, \underline{\alpha}_{V}, \bar{\alpha}_{V}, \underline{\alpha}_{W}, \bar{\alpha}_{W} \in$ $\mathcal{K}_{\infty}, \varrho \in \mathcal{K}$ such that the following conditions holds:

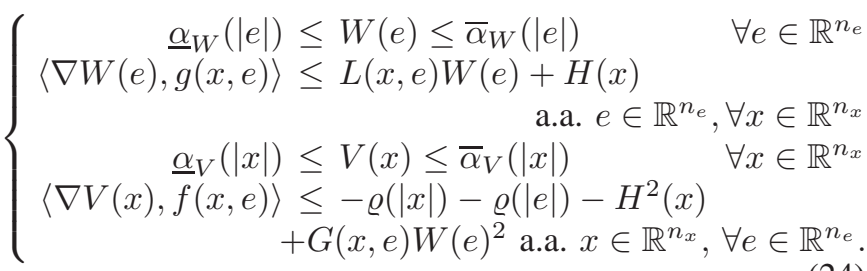

In [19], $L$ and $G$ are supposed to be constant that implies that system $\dot{x}=f(x, e)$ is $\mathcal{L}_{2}$-gain stable from $W$ to $H$. Making $L$ and $G$ state-dependent allows us to enlarge the studied class of systems and to eventually obtain less conservative upper bounds in the second and the fourth inequalities of Assumption 3 that will help to enlarge the inter-event intervals. Note that Assumption 3 does not necessarily imply that the system $\dot{x}=f(x, e)$ is ISS with respect to $e$. An example of nonlinear systems which verifies Assumption 3 is given in Section VI-B. Model (2) becomes here

$$
\left.\begin{array}{l}
\dot{x}=f(x, e) \\
\dot{e}=g(x, e) \\
\dot{\eta}=-2 \eta L(x, e)-\eta^{2}-G(x, e)
\end{array}\right\} q \in C
$$

where $q=(x, e, \eta), 0<\underline{c}<\bar{c}$ are design parameters and $\eta$ plays the role of $\zeta$ mentioned above and is called a clock 
variable. The sets $C$ and $D$ are

$$
C=\{q: \eta \in[\underline{c}, \bar{c}]\} \quad D=\{q: \eta=\underline{c}\} .
$$

Note that, instead of letting $\eta$ to decrease from $\rho^{-1}$ to $\rho$ like in [19], we consider any constants $0<\underline{c}<\bar{c}$. This gives more flexibility and may help generating larger interexecution intervals. The following theorem ensures the stability of system (25) and the existence of uniform semiglobal dwell-times. It follows from Theorem 1 using the Lyapunov function $R(q)=V(x)+\eta W(e)^{2}$.

Theorem 3: Consider system (25), (26) and suppose Assumption 3 holds. The solutions to (25) have a uniform semiglobal dwell-time and the set $\mathcal{A}=\{q:(x, e)=$ 0 and $\eta \in[\underline{c}, \bar{c}]\}$ is UGAS .

Remark 4: Suppose that $L(x, e) \leq \bar{L}$ and $G(x, e) \leq \bar{G}$ for any $(x, e) \in \mathbb{R}^{n_{x}+n_{e}}$ and some $\bar{L} \geq 0, \bar{G} \geq 1$. Then, the event-triggering condition in (26) will generate longer intertransmission intervals than the corresponding time-triggered one in [19] with $L=\bar{L}$ and $\gamma^{2}=\bar{G}$ in (9), (10) in [19], respectively. Indeed, transmissions are triggered in [19] when the solution to $\dot{\zeta}=-2 L \zeta-\gamma\left(\zeta^{2}+1\right)$ with $\zeta(0)=\rho^{-1}$ is equal to $\rho$ for some $\rho \in(0,1)$. By selecting $\bar{c}=\rho^{-1}$ and $\underline{c}=\rho$, we deduce from the comparison principle that $\zeta$ will be equal to $\rho^{-1}$ before $\eta$ becomes equal to $\underline{c}$, which results in smaller inter-transmission intervals for the time-triggering rule.

\section{Strategy in [28]}

The system $\dot{x}=f(x, e)$ is assumed to be input-to-state stable (ISS) with respect to the sampling-induced errors. The model (2) is here

$$
\left.\left.\begin{array}{l}
\dot{x}=f(x, e) \\
\dot{e}=g(x, e)
\end{array}\right\} q \in C, \quad \begin{array}{l}
x^{+}=x \\
e^{+}=0
\end{array}\right\} q \in D,
$$

where $q=(x, e)$. From (16), (17), we have that $V(x) \geq W(e)$ implies

$$
\langle\nabla V(x), f(x, e)\rangle \leq-(\mathbb{I}-\sigma) \circ \alpha(V(x)),
$$

The triggering rule in [28] corresponds to $W(e) \geq V(x)$ and leads to the following flow and jump sets for (27)

$$
C=\{q: W(e) \leq V(x)\}, \quad D=\{q: W(e) \geq V(x)\} .
$$

The following result is derived by applying Theorem 1 with $R(q)=\max \{V(x), W(e)\}$. The proof is omitted as it follows similar lines as the proof of Theorem 2.

Theorem 4: Consider system (27), (29), let $\mathcal{A}=\{q$ : $(x, e)=0\}$ and suppose Assumptions 1-2 hold with $W$ is continuously differentiable ${ }^{2}$. The solutions to (27) have a uniform semiglobal dwell-time outside $\mathcal{A}$ and $\mathcal{A}$ is UGAS. $\square$

When the controller is implemented using zero-order-hold devices as in [28], $g(x, e)=-f(x, e)$ in (27) and the third condition of Assumption 2 coincides with the first one in (23). Theorem 1 can also be used to analyse the implementation of the controller using the model-based technique proposed in [15]. In this setup, the control input applied to the plant is

\footnotetext{
${ }^{2}$ See Remark 2.
}

based on an estimate of the plant state between two successive transmissions, which is generated using a copy of the plant dynamics. Assume the control law applied to the plant dynamics $\dot{x}_{p}=f_{p}\left(x_{p}, u\right)$ is static, i.e. $u=g_{c}\left(\hat{x}_{p}\right)$. In this case, $\dot{\hat{x}}_{p}=$ $f_{p}\left(\hat{x}_{p}, g_{c}\left(\hat{x}_{p}\right)\right)$ on flows and $\hat{x}_{p}^{+}=x_{p}$ at jumps. Recalling that $x=x_{p}$ and $e=\hat{x}_{p}-x_{p}$ in this case, we obtain the model (27) with $f(x, e)=f_{p}\left(x_{p}, g_{c}\left(\hat{x}_{p}\right)\right)=f_{p}\left(x_{p}, g_{c}\left(x_{p}+e\right)\right)$ and $g(x, e)=f_{p}\left(\hat{x}_{p}, g_{c}\left(\hat{x}_{p}\right)\right)-f_{p}\left(x_{p}, g_{c}\left(\hat{x}_{p}\right)\right)=f_{p}\left(x_{p}+e, g_{c}\left(x_{p}+\right.\right.$ $e))-f_{p}\left(x_{p}, g_{c}\left(x_{p}+e\right)\right)$. The conclusions of Theorem 4 then apply when $f_{p}$ and $g_{c}$ are locally Lipschitz and are equal to zero at the origin. We make this observation about the modelbased approach explicit for this event-triggering technique, but it can also be done for the other strategies studied in this paper.

Remark 5: The condition $W(e) \leq V(x)$ is selected to ensure that $V$ always decreases. We could thus have defined the sets $C$ and $D$ for system (27) as in [6], [26]: $C=\{q:\langle\nabla V(x), f(x, e)\rangle \leq-(\mathbb{I}-\sigma) \circ \alpha(V(x))\}$, $D=\{q:\langle\nabla V(x), f(x, e)\rangle \geq-(\mathbb{I}-\sigma) \circ \alpha(V(x))\}$. The conclusions of Theorem 4 immediately apply in this case.

Remark 6: We explain how Proposition 2 can be used to relax the assumptions of Theorem 4 at the price of a weaker stability property. Instead of the first inequality in (23), suppose that for any $\mu>0$ and any compact $S \subset \mathbb{R}^{n_{x}+n_{e}}$, there exists $L_{1} \in \mathbb{R}_{\geq 0}$ such that for any $(x, e) \in S,|f(x, e)| \leq$ $L_{1}(|x|+|e|)+\mu$. This is the case for instance when $f$ is equal to zero at the origin and is locally Lipschitz everywhere except at zero. We can apply Proposition 2 to conclude that the set $R_{<}^{-1}(\varepsilon)$ is UGAS, where $\varepsilon=\bar{\alpha}_{R}(\theta \mu)$ for any $\theta>0$ and $\bar{\alpha}_{R}$ comes from the satisfaction of item (i) of Theorem 1 for $R(q)=\max \{V(x), W(e)\}$. Indeed, let $\mu=\theta^{-1} \bar{\alpha}_{R}^{-1}(\varepsilon)>0$, $S \subset \mathbb{R}^{n_{x}+n_{e}}$ be compact and fix $L_{1} \in \mathbb{R}_{>0}$. Let $(x, e) \in$ $S \cap R_{\geq}^{-1}(\varepsilon),|f(x, e)| \leq L_{1}(|x|+|e|)+\theta^{-1} \bar{\alpha}_{R}^{-1}(\varepsilon) \leq L_{1}(|x|+$ $|e|)+\bar{\theta}^{-1} \bar{\alpha}_{R}^{-1}(R(q)) \leq L_{1}(|x|+|e|)+\theta^{-1} \bar{\alpha}_{R}^{-1}\left(\bar{\alpha}_{R}(|(x, e)|)\right)$. Hence $|f(x, e)| \leq L_{1}(|x|+|e|)+\theta^{-1}|(x, e)| \leq\left(L_{1}+\right.$ $\left.\theta^{-1}\right)(|x|+|e|)$. We use Proposition 1 with $\psi(q)=\frac{|e|}{|x|}$ when $M|e| \leq|x|$ and $\psi(q)=M^{-1}$ otherwise for some $M>0$ (which is well-defined and locally Lipschitz on an open set containing $R_{\leq}^{-1}(\Delta) \backslash R_{\leq}^{-1}(\varepsilon)$ ) like in the proof of Theorem 2, to derive that the solutions to (27) with the flow and jump sets modified as in (13) have a uniform semiglobal dwell-time outside $R_{\leq}^{-1}(\varepsilon)$. We then apply Proposition 2 to obtain the desired result. It is interesting to note that the set $R_{<}^{-1}(\varepsilon)$ can be rendered as small as desired by decreasing $\vartheta$ in this case (at the price of shorter minimum inter-transmission times). $\square$

\section{Strategies in [13], [16], [20]}

We suppose that Assumption 1 is satisfied. The idea is to constrain the error $e$ to remain less than a given constant. In that way, transmissions are triggered whenever the condition below is satisfied

$$
\gamma(|e|) \geq \rho
$$

where $\rho>0$ is a design parameter and $\gamma$ is defined in Assumption 1. We obtain the following hybrid model

$$
\left.\left.\begin{array}{rl}
\dot{x} & =f(x, e) \\
\dot{e} & =g(x, e)
\end{array}\right\} \quad q \in C, \quad \begin{array}{l}
x^{+}=x \\
e^{+}=0
\end{array}\right\} \quad q \in D,
$$


where $q=(x, e)$ and

$$
C=\{q: \gamma(|e|) \leq \rho\} \quad D=\{q: \gamma(|e|) \geq \rho\} .
$$

The following result is an application of Theorem 1 with $R(q)=\max \left\{V(x)-\alpha^{-1}(2 \rho), 0\right\}+\max \{\gamma(|e|)-\rho, 0\}$ where $\alpha$ comes from Assumption 1.

Theorem 5: Consider system (31), (32) and suppose Assumption 1 holds and $e \mapsto \gamma(|e|)$ is continuously differentiable. The solutions to (31) have a uniform semiglobal dwelltime and the set $\mathcal{A}=\left\{q: V(x) \leq \alpha^{-1}(2 \rho)\right.$ and $\left.\gamma(|e|) \leq \rho\right\}$ is UGAS.

\section{E. Strategy in [34]}

The main idea of [34] is to construct the event-triggering condition such that a known Lyapunov function $V$ for the closed-loop system in the absence of sampling remains below a designed threshold which decreases to the origin. In that way, the convergence of the plant state to the origin is immediately guaranteed and the function $V$ is not forced to decrease all the time contrary to [28], which may reduce the need for control updates. We revisit this idea by introducing a variable to model the threshold.

We assume that the controller is designed to ensure Assumption 1 . The threshold variable is denoted by $\eta \in \mathbb{R}_{\geq 0}$ and it has the following dynamics on flows

$$
\dot{\eta}=-\delta(\eta)
$$

where $\delta$ is a designed continuous and positive definite function such that $\delta(s) \leq \sigma \circ \alpha(s)$ for any $s \geq 0$, with $\alpha$ is defined in Assumption 1 and $\sigma$ is any class- $\mathcal{K}_{\infty}$ function such that $\sigma(s)<s$ for any $s>0$. The selection of $\delta$ depends on the desired performances in terms of the convergence of the Lyapunov function $V$. The dynamics in (33) generalizes the corresponding one in [34] as the threshold is only required to asymptotically (and not necessarily exponentially) decrease to the origin. At jumps, $\eta$ takes the value of $V(x)$, i.e.

$$
\eta^{+}=V(x) \text {. }
$$

Following [34], the triggering condition is designed such that $V(x)$ is always below $\eta$ (except potentially at the initial time instant) which leads to the hybrid model below

$$
\left.\left.\begin{array}{l}
\dot{x}=f(x, e) \\
\dot{e}=g(x, e) \\
\dot{\eta}=-\delta(\eta)
\end{array}\right\} q \in C, \quad \begin{array}{l}
x^{+}=x \\
e^{+}=0 \\
\eta^{+}=V(x)
\end{array}\right\} q \in D,
$$

where $q=(x, e, \eta)$ and

$$
\begin{aligned}
& C=\{q: V(x) \leq \eta, \eta \geq 0\}, \\
& D=\{q: V(x) \geq \eta, \eta \geq 0,\langle\nabla V(x), f(x, e)\rangle \geq-\alpha(V(x))\} .
\end{aligned}
$$

The condition $\langle\nabla V(x), f(x, e)\rangle \geq-\alpha(V(x))$ is needed in the definition of $D$ to avoid the Zeno behaviour. Indeed, after a jump $\eta=V(x)$ but it is not necessary to jump again since $V(x)$ will decrease faster than $\eta$ in view of Assumption 1 and the way $\delta$ is designed in (33). The theorem below is an application of Theorem 1 with $R(q)=\max \{V(x), \eta\}$.

Theorem 6: Consider system (35), (36), let $\mathcal{A}=\{q$ : $(x, \eta)=0\}$, and suppose Assumptions 1-2 hold. The solutions to (35) have a uniform semiglobal dwell-time outside the origin and $\mathcal{A}$ is UGpAS.

Remark 7: The satisfaction of Assumption 2.1-2.2 in [34] implies the satisfaction ${ }^{3}$ of Assumptions 1-2 (when using zero-order-hold devices) but the opposite is not true. Hence Theorem 6 relies on weaker conditions than Theorem 3.3 in [34] (when task delays are ignored); see Section VI-A for an example where the Lyapunov function $V$ is quadratic which is not allowed by [34].

Remark 8: Similar results can be obtained for the policy proposed in [33], see Section V.A in [23].

\section{ILLUSTRATIVE EXAMPLES}

We compare the event-triggering techniques presented in Sections V-A-V-B with those in Sections V-C and V-E on two physical examples.

\section{A. Jet engine compressor}

We borrow the model from [14]: $\dot{x}_{1}=-x_{2}-\frac{3}{2} x_{1}^{2}-\frac{1}{2} x_{1}^{3}$, $\dot{x}_{2}=u$, where $x_{1}$ represents the mass flow, $x_{2}$ is the pressure rise and $u$ is the throttle mass flow. In this model the origin has been translated to the desired equilibrium point, and the objective is to steer $\left(x_{1}, x_{2}\right)$ to zero. The control law $u=4 x_{1}-4 x_{2}-\frac{9}{2} x_{1}^{2}-\frac{3}{2} x_{1}^{3}$ is designed to stabilize the origin. This controller is then implemented using zero-orderhold devices and is connected to the two sensors measuring $x_{1}$ and $x_{2}$ through a network. The objective is to stabilize the system while limiting the number of control inputs in order to reduce the usage of the communication channel (and not necessarily of the processor time) as well the actuator energy consumption. Thus, it is more convenient to work with the sampling-induced error on the control input $u$, i.e. $e=\hat{u}-u$, which means that we compute all the time $u$ and trigger transmission according to the selected event-triggering policy. The system under sampling-induced constraints is

$$
\dot{x}_{1}=-x_{2}-\frac{3}{2} x_{1}^{2}-\frac{1}{2} x_{1}^{3}, \quad \dot{x}_{2}=u+e .
$$

We want to compare the results given by the strategy presented in Section V-A and those obtained with [28] and [34]. We first need to verify that the required conditions are satisfied. Assumption 1 holds with ${ }^{4} V(x)=\frac{1}{2} x_{1}^{2}+\frac{1}{2}\left(x_{2}-3 x_{1}\right)^{2}$, $\underline{\alpha}_{V}(s)=0.045 s^{2}, \bar{\alpha}_{V}(s)=5.455 s^{2}, \alpha(s)=2 s, \gamma(s)=$ $5.2591 s^{6}+3.5797 s^{4}-0.0032626 s^{3}+4.6086 s^{2}$ for $s \geq 0$. We have obtained ${ }^{4} W(e)=1.62^{-1} e^{2}$ with $\sigma(s)=0.9 s$ for $s \geq 0$ which is directly computed to ensure (16). Assumption 2 holds as $f$ and $\alpha^{-1} \circ W$ are locally Lipschitz and are equal to zero at the origin. As a consequence, the conditions of Theorems 2, 4 and 6 are verified (as $W$ is continuously differentiable). We can therefore apply the three corresponding event-triggering policies. It has to be noted that we cannot apply the technique in [34] as the conditions (7) and (9) in [34] are not satisfied.

\footnotetext{
${ }^{3}$ Assumption 1 generalizes Assumption 2.1 in [34]. Equation (6) in [34] corresponds to the first inequality in (23) and (7)-(9) in [34] ensure the second inequality in (23) holds; noting that $\bar{\alpha} \circ \alpha_{2}^{-1}(s) \geq \bar{a} s$ for $s \geq 0$ is used in the proof of Theorem 3.3 in [34] instead of (9) in [34], which guarantees that the corresponding function $\alpha^{-1}$ in (15) is locally Lipschitz.

${ }^{4}$ SOSTools ([25]) was used to compute $\alpha, \gamma$ and $W$.
} 
We therefore consider the results in Section V-E. We take the same scalar field $\delta$ for the strategies of Sections V-A and V-E, namely $\delta(s)=\sigma \circ \alpha(s)=1.8 s$ for $s \geq 0$.

We consider 100 initial conditions for the $x$-system, which are uniformly distributed on the circle centered at the origin and of radius 10 . We compare the average inter-transmission times generated by each event-triggering condition for a simulation time of 100 seconds in Table I and for the time it takes for $V(x)$ to become less than $0.01 V(x(0,0))$, which we denote $T^{\star}$, in Table II. We see that the strategy in Section V-A can lead to less transmissions and that playing with $\eta(0,0)$ allows to trade performance (i.e. time $T^{*}$ in this case) for the number of transmissions.

It has to be emphasized that the results obtained in this section depend on the considered Lyapunov function $V$ and on the parameters of the event-triggering mechanisms. Moreover, each of the presented event-triggering techniques ensures a different asymptotic stability property. That is the reason why we cannot assess whether one method is better than another in general. Nevertheless, we have seen that the proposed technique in Section V-A exhibits great potential for real-time scheduling, since its parameters can be designed according to the available resources. For instance, functions $\delta$ in (18) with slow increasing slopes could be chosen in case of overload in the network or in the processor executing the controller for example.

\section{B. van der Pol oscillator}

We consider the forced van der Pol oscillator: $\dot{x}_{1}=x_{2}$, $\dot{x}_{2}=\left(1-x_{1}^{2}\right) x_{2}-x_{1}+u$, where $x=\left(x_{1}, x_{2}\right) \in \mathbb{R}^{2}$ is the state and $u$ is the control input. The controller $u=-x_{2}-\left(1-x_{1}^{2}\right) x_{2}$ is designed to exponentially stabilize the origin of the system. We envision the scenario where a network is used to close the feedback loop. As in Section VI-A, we consider the error induced on the control input $e=\hat{u}-u$, we thus obtain the system below

$$
\dot{x}_{1}=x_{2}, \quad \dot{x}_{2}=-x_{1}-x_{2}+e .
$$

We either use the event-triggering policy of Section V-B or the one ${ }^{5}$ in [28]. We first need to verify that the required conditions are satisfied. For the technique in Section V-B, Assumption 3 is ensured with $6(x)=1.3815 x_{1}^{6}+$ $1.4349 x_{1}^{5} x_{2}+3.6869 x_{1}^{4} x_{2}^{2}+1.928 x_{1}^{3} x_{2}^{3}+4.7197 x_{1}^{2} x_{2}^{4}+$ $0.83432 x_{1}^{2}+4.054 x_{1} x_{2}^{5}-1.9823 x_{1} x_{2}+2.2712 x_{2}^{6}+3.4733 x_{2}^{2}$, $\underline{\alpha}_{V}(s)=0.5 s^{2}, \bar{\alpha}_{V}(s)=10\left(s^{2}+s^{6}\right), W(e)=|e|$, $\underline{\alpha}_{W}(s)=\bar{\alpha}_{W}(s)=s, L(x, e)=\left|x_{1}^{2}-2\right|, H(x)=$ $\left|-x_{1}^{3}-x_{1}^{2} x_{2}+2 x_{1} x_{2}^{2}+2 x_{1}+2 x_{2}\right|, G(x, e)=8.001$ and $\varrho(s)=0.001 s$ for $s \geq 0$. In that way, the conditions of Theorem 3 are verified. We have heuristically selected $\underline{c}$ small, $\underline{c}=0.001$, and we take different values for $\bar{c}$ in order to study its impact on the amount of transmissions.

\footnotetext{
${ }^{5}$ We do not compare the results with those given by the event-triggering technique in Section V-E, because different Lyapunov functions will be designed to verify Assumptions 1 and 3, adding to this the flexibility of the triggering condition in Section V-E in terms of the choice of the dynamics of $\eta$ and its initial condition render fair comparisons difficult.

${ }^{6}$ SOSTools ([25]) was used to compute $V, W, L, G, \varrho, \gamma, \alpha$.
}

\begin{tabular}{cccc} 
[28] & \multicolumn{3}{c}{ Section V-B } \\
& $\bar{c}=1$ & $\bar{c}=10$ & $\bar{c}=1000$ \\
\hline 0.0350 & 0.0622 & 0.0727 & 0.0847 \\
\hline
\end{tabular}

TABLE III

AVERAGE INTER-TRANSMISSION TIME FOR 50 INITIAL CONDITIONS FOR A SIMULATION TIME OF $5 \mathrm{~s}$.

To verify Assumption 1, we choose not to use the same Lyapunov function as above because it would typically lead to conservative estimates of $\alpha$ and $\gamma$ in (15), which would give rise to many transmissions. Instead, we take $V$ to be $V(x)=0.0058679 x_{1}^{2}+0.0040791 x_{1} x_{2}+0.0063684 x_{2}^{2}$ for the strategy in [28] which ensures Assumption 1 with $\alpha(s)=$ $0.5 s, \gamma(s)=0.01 s^{2}$ for $s \geq 0$. The function $W$ in (17) is different from the one used in Assumption 3: $W(e)=2.222 e^{2}$ for $\sigma(s)=0.9 s$ for $s \geq 0$. In that way, the conditions of Theorem 4 are verified.

The obtained average inter-transmissions times for each strategies are summarized in Table III, where 50 initial conditions for the $x$-system have been taken uniformly on the circle of the center the origin and of radius 10 . We notice that the policy in Section V-B may allow to reduce the usage of the network by adjusting the parameter $\bar{c}$. It has to be noted that we could not compare these results with the time-triggered setup of [19] as we were not able to show that the required conditions hold.

\section{CONCLUSION}

We have investigated the event-triggered stabilization of nonlinear systems using the hybrid formalism of [8]. This approach allows to capture in a unified manner many eventtriggering conditions available in the literature [13], [16], [20], [28], [34]. Moreover, two new policies have been presented which both make use of additional variables. They have been shown to potentially reduce the amount of transmissions compared to [28], [34] on two physical examples. All the studied strategies are guaranteed to ensure the existence of a uniform minimum amount of time between any two jumps.

The envisioned setup requires the continuous evaluation of the triggering condition. In practice, the triggering mechanism is typically implemented on a digital platform, hence the transmission criterion is only periodically evaluated, leading to a periodic event-triggered control paradigm, see, e.g., [1], [10]. The results of this paper can be combined with those in [22] to derive triggering strategies which take into account the hardware sampling. On the other hand, throughout the paper, we have focused on the so-called one packet transmission problem as all the states are sent together in a single packet. This generally implies the collocation of all sensors and, for multiple-input control systems, collocation of all actuators as well. The extension of this work to distributed event-triggered control would be an interesting direction to follow. Preliminary results are presented in [24].

\section{ACKNOWLEDGMENT}

Romain Postoyan would like to thank Luca Zaccarian for his help on Theorem 1 and Constantin Morărescu for fruitful discussions. 


\begin{tabular}{ccccc} 
& Section V-A & [28] & Section V-E \\
$\eta(0,0)=V(x(0,0))$ & $\eta(0,0)=\max _{|x| \leq 10} V(x)$ & $\eta(0,0)=10^{4}$ & & \\
\hline 0.5472 & 0.5645 & 0.6199 & 0.5030 & 0.4750 \\
\hline
\end{tabular}

TABLE I

AVERAGE INTER-TRANSMISSION TIME FOR A SIMULATION TIME OF 100S.

\begin{tabular}{cccccc} 
& \multicolumn{1}{c}{ Section V-A } & [28] & Section V-E \\
& $\eta(0,0)=V(x(0,0))$ & $\eta(0,0)=\max _{|x| \leq 10} V(x)$ & $\eta(0,0)=10^{4}$ & & \\
\hline$T^{*}$ & 1.7563 & 1.9805 & 2.0103 & 1.8549 & 1.0572 \\
Number of transmissions & 32.02 & 19.87 & 7.68 & 34.32 & 12.37 \\
\hline
\end{tabular}

TABLE II

AVERAGE VALUES OF $T^{*}$ AND OF THE NUMBER OF TRANSMISSIONS.

\section{APPENDIX}

Proof of Lemma 3. We only consider the case where $\mathcal{O}$ is non-empty, closed and unbounded. The proof follows similar lines when $\mathcal{O}$ is non-empty and compact. Let $\psi: s \mapsto \inf _{|x|_{\mathcal{A}} \geq s, x \in \mathcal{O}} V(x)$ which is well-defined on $\mathbb{R}_{\geq 0}$. Indeed, the set $\left\{x \in \mathcal{O}:|x|_{\mathcal{A}} \geq s\right\}$ is non-empty and closed for any $s \geq 0$ (as $\mathcal{A}$ is a compact subset of the unbounded set $\mathcal{O}$ ), furthermore $V$ is non-negative and continuous. The function $\psi$ is positive definite, non-decreasing and grows unbounded as $s \rightarrow \infty$ in view of the assumptions on $V$. Furthermore, for any $x \in \mathcal{O}, \psi\left(|x|_{\mathcal{A}}\right) \leq V(x)$. The function $\psi$ is a priori neither continuous nor strictly increasing, however we can always construct $\alpha_{1} \in \mathcal{K}_{\infty}$ such that $\alpha_{1}(s) \leq \psi(s)$ for any $s \geq 0$. Let $\chi: s \mapsto \sup _{|x|_{\mathcal{A}} \leq s, x \in \mathcal{O}} V(x)$. Like above, this function is well-defined on $\mathbb{R}_{\geq 0}$, non-decreasing, grows unbounded and such that $V(x) \leq \chi\left(|x|_{\mathcal{A}}\right)$ for any $x \in \mathcal{O}$. We need to ensure that $\chi$ is continuous at 0 in order to be able to upper-bound it by a class- $\mathcal{K}_{\infty}$ function $\alpha_{2}$. Let $\varepsilon>0$, there exists $\delta \geq 0$ such that $|x-y| \leq \delta$ implies $|V(x)-V(y)| \leq \varepsilon$ by continuity of $V$. Let $\eta \in[0, \delta]$, $\chi(\eta)=\sup _{|x|_{\mathcal{A}}<\eta, x \in \mathcal{O}} V(x)=V(y)$ for some $y \in \mathcal{O}$ with $|y|_{\mathcal{A}} \leq \eta$, as $V$ is continuous and the set $\left\{x \in \mathcal{O}:|x|_{\mathcal{A}} \leq \eta\right\}$ is compact. Because $\mathcal{A}$ is closed, there exists $z \in \mathcal{A}$ such that $|y-z|=|y|_{\mathcal{A}} \leq \eta \leq \delta$. By continuity of $V$, $V(y)-V(z)=V(y)-0 \leq \varepsilon$. Hence $\chi(\eta) \leq \varepsilon$ for any $\eta \in[0, \delta]$ which proves the continuity of $\chi$ at 0 . As a consequence, we can always construct $\alpha_{2} \in \mathcal{K}_{\infty}$ which upper-bounds $\chi$.

Proof of Lemma 4. Let $x \in C \cap D$ with $f(x) \in T_{C}(x)$. According to the definition of the tangent cone (see Definition 1), there exist sequences $\left\{x_{i}\right\}_{i \in \mathbb{Z}_{\geq 0}}$ and $\left\{\tau_{i}\right\}_{i \in \mathbb{Z}_{\geq 0}}$ with $x_{i} \in$ $C$ and $\tau_{i}>0$ for any $i \in \mathbb{Z}_{\geq 0}$ and $x_{i} \rightarrow x, \tau_{i} \rightarrow 0$ as $i \rightarrow \infty$, such that $f(x)=\lim _{i \rightarrow \infty}\left(x_{i}-x\right) / \tau_{i}$. On the other hand,

$$
\begin{aligned}
& \langle\nabla V(x), f(x)\rangle-\langle\nabla W(x), f(x)\rangle \\
& \quad=\lim _{h \rightarrow 0} \frac{V(x+h f(x))-V(x)-W(x+h f(x))+W(x)}{h}
\end{aligned}
$$

which simplifies to, as $V(x)=W(x)$ since $x \in C \cap D$,

$$
\begin{aligned}
& \langle\nabla V(x), f(x)\rangle-\langle\nabla W(x), f(x)\rangle \\
& \quad=\lim _{h \rightarrow 0} \frac{V(x+h f(x))-W(x+h f(x))}{h} .
\end{aligned}
$$

We proceed by contradiction and suppose that $\lim _{h \rightarrow 0} \frac{V(x+h f(x))-W(x+h f(x))}{h}<0$. Since $\tau_{i}>0$ and $\tau_{i} \rightarrow 0$ as $i \rightarrow \infty$,

$$
\begin{aligned}
\lim _{h \rightarrow 0} & \frac{V(x+h f(x))-W(x+h f(x))}{h} \\
= & \lim _{i \rightarrow \infty} \frac{V\left(x+\tau_{i} f(x)\right)-W\left(x+\tau_{i} f(x)\right)}{\tau_{i}} \\
= & \lim _{i \rightarrow \infty}\left(\frac{V\left(x+\tau_{i} f(x)\right)-W\left(x+\tau_{i} f(x)\right)}{\tau_{i}}\right. \\
& \left.+\frac{V\left(x_{i}\right)-W\left(x_{i}\right)}{\tau_{i}}-\frac{V\left(x_{i}\right)-W\left(x_{i}\right)}{\tau_{i}}\right) \\
= & \lim _{i \rightarrow \infty} \frac{V\left(x_{i}\right)-W\left(x_{i}\right)}{\tau_{i}} \\
& +\lim _{i \rightarrow \infty} \frac{V\left(x+\tau_{i} f(x)\right)-V\left(x_{i}\right)}{\tau_{i}} \\
& -\lim _{i \rightarrow \infty} \frac{W\left(x+\tau_{i} f(x)\right)-W\left(x_{i}\right)}{\tau_{i}}
\end{aligned}
$$

where $x_{i}, i \in \mathbb{Z}_{\geq 0}$ are defined above. Since $V$ is continuously differentiable, it is locally Lipschitz. Consequently, there exists $L \geq 0$ such that

$$
\begin{aligned}
\lim _{i \rightarrow \infty}\left|\frac{V\left(x+\tau_{i} f(x)\right)-V\left(x_{i}\right)}{\tau_{i}}\right| & \leq \lim _{i \rightarrow \infty} L \frac{\left|x-x_{i}+\tau_{i} f(x)\right|}{\tau_{i}} \\
& =\lim _{i \rightarrow \infty} L\left|\frac{x-x_{i}}{\tau_{i}}+f(x)\right| \\
& =0
\end{aligned}
$$

since $f(x)=\lim _{i \rightarrow \infty}\left(x_{i}-x\right) / \tau_{i}$. By applying the same arguments on $\lim _{i \rightarrow \infty} \frac{W\left(x+\tau_{i} f(x)\right)-W\left(x_{i}\right)}{\tau_{i}}$, we derive from (41) that

$$
\langle\nabla V(x), f(x)\rangle-\langle\nabla W(x), f(x)\rangle=\lim _{i \rightarrow \infty} \frac{V\left(x_{i}\right)-W\left(x_{i}\right)}{\tau_{i}} \text {. } 43 \text {. }
$$

Since $x_{i} \in C$ for any $i \in \mathbb{Z}_{\geq 0}, V\left(x_{i}\right) \geq W\left(x_{i}\right)$. Hence

$$
\begin{aligned}
0 & \leq \lim _{i \rightarrow \infty} \frac{V\left(x_{i}\right)-W\left(x_{i}\right)}{\tau_{i}} \\
& \leq \lim _{h \rightarrow 0} \frac{V(x+h f(x))-W(x+h f(x))}{h}
\end{aligned}
$$

which contradicts $\lim _{h \rightarrow 0} \frac{V(x+h f(x))-W(x+h f(x))}{h}<0$. Hence $\langle\nabla V(x), f(x)\rangle \geq\langle\nabla W(x), f(x)\rangle$ in view of (40) which concludes the proof.

We need the lemma below in the proof of Theorem 1 .

Lemma 5: Let $\phi$ be a solution to (2), it holds that $\frac{d}{d t} \phi(t, j) \in\{F(\phi(t, j))\} \cap T_{C}(\phi(t, j))$ for all $j \in \mathbb{Z}_{\geq 0}$ and almost all $t \in I^{j}$, where $I^{j}=\{t:(t, j) \in \operatorname{dom} \phi\}$.

Proof. The proof follows the same lines as the proof of the necessity of (a) in Lemma 5.26 in [8], which we recall. Since $\phi$ is a solution to (2) and $C$ is closed, $\phi(t, j) \in C$ for all $j \in \mathbb{Z}_{\geq 0}$ and $t \in I^{j}$. Furthermore $\frac{d}{d t} \phi(t, j)$ exists and belongs to 
$\{F(\phi(t, j))\}$ for all $j \in \mathbb{Z}_{\geq 0}$ and almost all $t \in I^{j}$ according to (6.1) in [8] (note that system (2) satisfies Assumption 6.5 in [8]). Hence for any $j \in \mathbb{Z}_{\geq 0}$ and almost all $t \in I^{j}$, for any sequence $\left\{t_{i}\right\}_{i \in \mathbb{Z}_{>0}}$ with $t_{i}>0, t_{i} \rightarrow 0$ as $i \rightarrow \infty$, and $t+t_{i} \in I^{j}, \frac{d}{d t} \phi(t, j)=\lim _{i \rightarrow \infty}\left(\phi\left(t+t_{i}, j\right)-\phi(t, j)\right) / t_{i}$. By definition of the tangent cone (see Definition 1), as $\phi\left(t+t_{i}, j\right) \in C$ for any $i \in \mathbb{Z}_{>0}$ (as $t+t_{i} \in I^{j}$ ), $\lim _{i \rightarrow \infty}\left(\phi\left(t+t_{i}, j\right)-\phi(t, j)\right) / t_{i} \in T_{C}(\phi(t, j))$. Hence, we have proved that $\frac{d}{d t} \phi(t, j) \in\{F(\phi(t, j))\} \cap T_{C}(\phi(t, j))$ for all $j \in \mathbb{Z}_{\geq 0}$ and almost all $t \in I^{j}$.

Proof of Theorem 1. Let $\phi$ be a solution to (2), $(t, j) \in \operatorname{dom} \phi$ and $0=t_{0} \leq t_{1} \leq \ldots \leq t_{j+1}=t$ satisfy

$$
\operatorname{dom} \phi \cap([0, t] \times\{0, \ldots, j\})=\bigcup_{i \in\{0, \ldots, j\}}\left[t_{i}, t_{i+1}\right] \times\{i\} .
$$

For each $i \in\{0, \ldots, j\}$ and almost all $s \in\left[t_{i}, t_{i+1}\right], \phi(s, i) \in$ $C$ and $\frac{d}{d s} \phi(s, i) \in\{F(\phi(s, i))\} \cap T_{C}(\phi(s, i))$ (according to Lemma 5). Hence, (11) implies that, for each $i \in\{0, \ldots, j\}$ and for almost all $s \in\left[t_{i}, t_{i+1}\right]$,

$$
R^{\circ}\left(\phi(s, i) ; \frac{d}{d s} \phi(s, i)\right) \leq-\alpha_{R}(R(\phi(s, i))) .
$$

As a consequence, since $R$ is locally Lipschitz, it holds that (see p. 99 in [30]),

$$
\frac{d}{d s} R(\phi(s, i)) \leq R^{\circ}\left(\phi(s, i) ; \frac{d}{d s} \phi(s, i)\right) .
$$

In view of (46) and (47), for each $i \in\{0, \ldots, j\}$ and for almost all $s \in\left[t_{i}, t_{i+1}\right]$,

$$
\frac{d}{d s} R(\phi(s, i)) \leq-\alpha_{R}(R(\phi(s, i))) .
$$

We derive that the set $\mathcal{A}$ is uniformly globally stable by following the same lines as in the proof of Theorem 3.18 in [8]. To prove uniform global pre-attractivity, we follow similar arguments as in the proof of Proposition 3.27 in [8]. For this purpose, we show that the last condition of Proposition 3.27 in [8] is verified. Let $r>0$ and $\phi$ be a solution to (2) with $|\phi(0,0)|_{\mathcal{A}} \leq r$. Take $(t, j) \in \operatorname{dom} \phi$ with $t+j \geq T$ for some $T \geq 0$. If there exists $t^{\prime}+j^{\prime} \leq t+j$ such that $\phi\left(t^{\prime}, j^{\prime}\right) \in \mathcal{A}$, $\phi(t, j) \in \mathcal{A}$ since the set $\mathcal{A}=R^{-1}(0)$ is strongly forward pre-invariant (see Definition 2). Then the solution has reached the attractor $\mathcal{A}$. If it is not the case, then $t \geq \tau(r)(j-1)$, i.e. $\frac{t}{\tau(r)}+1 \geq j$, where $\tau(r)$ comes from Definition 3 according to item (iv) of Theorem 1. Therefore $t+j \geq T$ implies $t+\frac{t}{\tau(r)}+1 \geq T$. We deduce that $t \geq \gamma_{r}(T)-N_{r}$ with $\gamma_{r}(s)=\left(1+\frac{1}{\tau(r)}\right)^{-1} s$ for $s \geq 0$ which is of class $\mathcal{K}_{\infty}$ and $N_{r}=\left(1+\frac{1}{\tau(r)}\right)^{-1}$. We can thus invoked the same arguments as for the proof of Proposition 3.27 in [8] to conclude that $\mathcal{A}$ is UGpAS. When $\mathcal{A}$ is compact, the fact that it is UGpAS for system (2) prevents the existence of finite escape times. Hence, the conditions at the end of Theorem 1 allow us to apply Proposition 6.10 in [8] to conclude that any maximal solution is complete.

Proof of Proposition 1. Let $\Delta \geq 0$ and $\phi$ be a solution to (2) with $|\phi(0,0)|_{\mathcal{A}} \leq \Delta$. Since items (i)-(iii) of Theorem 1 hold, $R(\phi(t, j)) \leq \bar{\Delta}$ for any $(t, j) \in \operatorname{dom} \phi$ with $\bar{\Delta}=\bar{\alpha}_{R}(\Delta)$. Suppose there exist $0 \leq t_{1} \leq t_{2}$ such that $\left(t_{1}, 0\right),\left(t_{1}, 1\right) \in \operatorname{dom} \phi$ and $\left(t_{2}, 1\right),\left(t_{2}, 2\right) \in \operatorname{dom} \phi$; otherwise the solution never jumps or jumps only once and the desired result trivially holds. Suppose also that $\phi(t, 1) \notin \mathcal{A}$ for all $t \in\left[t_{1}, t_{2}\right]$; otherwise the solution enters in the attractor $\mathcal{A}$ and remains in it for all future times as this set is strongly forward pre-invariant for system (2) (see Definition 2) since it is closed and uniformly globally stable in view of the proof of Theorem 1. Hence $\phi(t, 1) \in \mathcal{S}(\bar{\Delta})$ for all $t \in\left[t_{1}, t_{2}\right]$. We also have that $\phi\left(t_{1}, 0\right)$ belongs to $R_{\leq}^{-1}(\bar{\Delta})$ and to $D$ (by definition of $t_{1}$ ), however it does not belong to $\mathcal{A}$ as otherwise $\phi\left(t_{1}, 1\right) \in \mathcal{A}$ by strong forward pre-invariance of $\mathcal{A}$. In other words, $\phi\left(t_{1}, 0\right) \in \mathcal{S}(\bar{\Delta}) \cap D$. Consider the function $\psi$ from item (ii) of Proposition 1 which is defined on an open set containing $\mathcal{S}(\bar{\Delta})$. Since $\phi\left(t_{1}, 0\right) \in \mathcal{S}(\bar{\Delta}) \cap D$ and $\phi\left(t_{1}, 1\right) \in \mathcal{S}(\bar{\Delta}), \psi\left(\phi\left(t_{1}, 1\right)\right) \leq a$ according to item (ii-a) of Proposition 1. By continuity of $t \mapsto \phi(t, 1)$ on $\left[t_{1}, t_{2}\right)$ and of $\psi$ on $\mathcal{S}(\bar{\Delta})$, we deduce that the next jump instant cannot occur before $\psi(\phi(t, 1))$ becomes equal to $b$ in view of item (ii-b) of Proposition 1. For almost all $t \in\left[t_{1}, t_{2}\right], \frac{d}{d t} \psi(\phi(t, 1)) \leq \lambda(\psi(\phi(t, 1)))$ in view of item (ii-c) of Proposition 1 and p.99-100 in [30]. Therefore, the next jump cannot occur before $\theta$, the solution to $\dot{\theta}=\lambda(\theta)$ with $\theta(0)=a$, becomes equal to $b$, in view of the standard comparison principle. We denote by $\tau(\Delta)$ this time which is strictly positive (note that $a, b, \lambda$ may depend on $\bar{\Delta}$ and thus on $\Delta)$. Hence we have proved that $t_{2}-t_{1}>\tau(\Delta)$. We deduce that the solutions to (2) have a uniform semiglobal dwell-time outside $\mathcal{A}$ by induction. Assume now that $\mathcal{A}$ compact and that item (ii) of Proposition 1 holds with $\mathcal{S}(\bar{\Delta})=R_{<}^{-1}(\bar{\Delta})$. Let $\Delta \geq 0$ and $\phi$ be a solution to (2) with $|\phi(0,0)| \leq \bar{\Delta}$. Since $\mathcal{A}$ is compact, there exists $\widetilde{\Delta} \geq 0$ such that $|\phi(0,0)|_{\mathcal{A}} \leq \widetilde{\Delta}$. It then suffices to follow similar lines as above to conclude that the solutions to (2) have a uniform semiglobal dwell-time in this case.

Proof of Proposition 2. Let $\widehat{R}: q \mapsto \max \{0, R(q)-\varepsilon\}$ which is well-defined on $\widehat{C} \cup \widehat{D} \cup G(\widehat{D}) \subseteq C \cup D \cup G(D)$. This function is non-negative, locally Lipschitz on an open set containing $\widehat{C}$ (thus continuous) and is equal to zero only on $\widehat{\mathcal{A}}$. Moreover, $\widehat{R}(q)$ tends to $\infty$ as $|q|_{\widehat{\mathcal{A}}}$ tends to $\infty$. Indeed, $|q|_{\widehat{\mathcal{A}}} \rightarrow \infty$ implies that $|q|_{\mathcal{A}} \rightarrow \infty$ since $|q|_{\widehat{\mathcal{A}}} \leq|q|_{\mathcal{A}}($ as $\mathcal{A} \subset \widehat{\mathcal{A}})$. We then use the left hand-side of item (i) of Theorem 1. Hence there exist $\hat{\alpha}_{1}, \hat{\alpha}_{2} \in \mathcal{K}_{\infty}$ such that $\hat{\alpha}_{1}\left(|q|_{\widehat{\mathcal{A}}}\right) \leq \widehat{R}(q) \leq \hat{\alpha}_{2}\left(|q|_{\widehat{\mathcal{A}}}\right)$ for any $q \in \widehat{C} \cup \widehat{D} \cup G(\widehat{D})$ in view of Lemma 3. Let $q \in \widehat{C}$ and $F(q) \in T_{\widehat{C}}(q)$. Consider the case where $R(q)-\varepsilon>0$, i.e. $q \in C \backslash R_{<}^{-1}(\varepsilon)$, then $T_{\widehat{C}}(q)=T_{C}(q)$ and thus $F(q) \in T_{C}(q)$. As a consequence, we use item (ii) of Theorem 1 (which is assumed to hold) to derive that $\widehat{R}^{\circ}(q ; F(q)) \leq-\alpha_{R}(R(q))=-\alpha_{R}(\widehat{R}(q)+\varepsilon) \leq-\alpha_{R}(\widehat{R}(q))$. If $R(q)-\varepsilon<0$, then $\widehat{R}^{\circ}(q ; F(q))=0$. Consider now the case where $R(q)-\varepsilon=0$ and note that $T_{\widehat{C}}(q)=T_{C}(q) \cup T_{R_{\leq}^{-1}(\varepsilon)}$. If $F(q) \in T_{C}(q)$, we deduce that $\widehat{R}^{\circ}(q ; F(q))=$ $\max \{\langle\nabla R(q), F(q)\rangle, 0\} \leq \max \left\{-\alpha_{R}(R(q)), 0\right\} \leq 0$ (using Lemma 1). If $F(q) \in T_{R_{\zeta}^{-1}(\varepsilon)}$, we apply Lemma 4 with $V(x)=\varepsilon$ and $W(x)=R(q)$ to derive $\langle\nabla R(q), F(q)\rangle \leq\langle\nabla \varepsilon, F(q)\rangle=0$. Hence, when $R(q)-\varepsilon=0$ 
and $F(q) \in T_{\widehat{C}}(q), \widehat{R}^{\circ}(q ; F(q)) \leq 0=-\alpha(\widehat{R}(q))$ (as $q \in \widehat{\mathcal{A}}$ in this case and $\widehat{R}$ is equal to zero on $\widehat{\mathcal{A}})$. We have shown that $\widehat{R}^{\circ}(q ; F(q)) \leq-\alpha(\widehat{R}(q))$ for any $q \in \widehat{C}$ with $F(q) \in T_{\widehat{C}}(q)$. Let $q \in D$, $\widehat{R}(G(q))=\max \{R(G(q))-\varepsilon, 0\} \leq \max \{R(q)-\varepsilon, 0\}$ in view of item (iii) of Theorem 1 . Hence $\widehat{R}(G(q)) \leq \widehat{R}(q)$. Finally, in view of item (ii) of Proposition 2, we apply the same arguments as in the proof of Theorem 1 to conclude that $\widehat{\mathcal{A}}$ is UGpAS. Noting that $G(\widehat{D}) \subset G(D) \subset C \cup D \subset \widehat{C} \cup \widehat{D}$, we follow the same lines as those in the proof of Theorem 1 to ensure that $\widehat{\mathcal{A}}$ is UGAS. The last part of Proposition 2 is obtained by using the function $R$ (instead of $\widehat{R}$ ), the fact that $R^{\circ}(q ; F(q)) \leq-\alpha_{R}(\varepsilon)$ when $q \in C, R(q) \geq \varepsilon$ and $F(q) \in T_{C}(q)$ and by following similar arguments as in the proof of Theorem 1.

Proof of Theorem 2. We consider the locally Lipschitz Lyapunov function, for all $q \in \mathbb{R}^{n_{q}}$,

$$
R(q)=\max \{V(x), W(e), \eta\} .
$$

Item (i) of Theorem 1 holds with $\mathcal{A}=\{0\}$ by using (14), Lemma 2 and noting that $\eta \geq 0$ on $C \cup$ $D \cup G(D)$. Let $q \in C$ and $F(q) \in T_{C}(q)$. When $R(q)=V(x)>\max \{W(e), \eta\}$, we derive from (16) and Lemma 1 that $R^{\circ}(q ; F(q))=\langle\nabla V(x), f(x, e)\rangle \leq$ $-(\mathbb{I}-\sigma) \circ \alpha(V(x))=-(\mathbb{I}-\sigma) \circ \alpha(R(q))$. Similarly, when $R(q)=\eta>\max \{V(x), W(e)\}, R^{\circ}(q ; F(q))=$ $-\delta(\eta)=-\delta(R(q))$. When $R(q)=\eta=V(x)>$ $W(e), R^{\circ}(q ; F(q))=\max \{\langle\nabla V(x), f(x, e)\rangle,\langle\nabla \eta,-\delta(\eta)\rangle\}$ according to Lemma 1 which is less than or equal to $-\min \{(\mathbb{I}-\sigma) \circ \alpha(R(q)), \delta(R(q))\}$. Consider the case where $R(q)=W(e)=\max \{\eta, V(x)\}$, i.e. $q \in C \cap D$. We write $C=C_{1} \cup C_{2}$ where $C_{1}=\{q: V(x) \geq W(e)\}$ and $C_{2}=\{q: \eta \geq W(e)\}$. When $q \in C_{1} \backslash C_{2}$, necessarily $F(q) \in T_{C_{1}}(q)$ and we again apply Lemma 1 to obtain $R^{\circ}(q ; F(q))=\max \{\langle\nabla V(x), f(x, e)\rangle,\langle\nabla W(e), g(x, e)\rangle\}$. Since $\langle\nabla V(x), f(x, e)\rangle \leq-(\mathbb{I}-\sigma) \circ \alpha(V(q))=-(\mathbb{I}-\sigma) \circ$ $\alpha(R(q))$ for any $q \in C_{1}$, by applying Lemma 4 , we derive that $R^{\circ}(q ; F(q))=\langle\nabla V(x), f(x, e)\rangle \leq-(\mathbb{I}-\sigma) \circ \alpha(R(q))$. A similar conclusion holds when $q \in C_{2} \backslash C_{1}$. Suppose now that $q \in C_{1} \cap C_{2} \cap D$. Since $F(q) \in T_{C}(q), F(q)$ belongs to $T_{C_{1}}(q)$ or to $T_{C_{2}}(q)$. Take $F(q) \in T_{C_{1}}(q)$ without loss of generality. We obtain that $R^{\circ}(q ; F(q)) \leq-(\mathbb{I}-\sigma) \circ \alpha(R(q))$ by invoking the same arguments as above. Consequently, item (ii) of Theorem 2 holds with $\alpha_{R}=\min \{(\mathbb{I}-\sigma) \circ \alpha, \delta\}$. Let $q \in D$,

$$
R(G(q))=\max \{V(x), 0, W(e)\} \leq R(q)
$$

so that item (iii) of Theorem 1 holds. We now show that item (iv) of Theorem 1 is satisfied using Proposition 1. Let $\Delta \geq 0$ and $\bar{\Delta}:=\bar{\alpha}_{R}(\Delta)$. We work on the set $R_{<}^{-1}(\bar{\Delta})$ which is compact and strongly forward pre-invariant (see Definition 2 ) as items (i)-(iii) of Theorem 1 hold. The inter-jump time is the time it takes for $W(e)$ to grow from 0 to $\max \{V(x), \eta\}$. We look for a lower bound on this time. For any $q \in R_{<}^{-1}(\bar{\Delta})$, $W(e) \leq M|e|$ and $\underline{\alpha}_{V}^{-1} \circ W(e) \leq M|e|$ for some $M>0$ as $W$ is continuously differentiable and Assumption 2 holds. Hence, the inter-jump time is lower bounded by the time it takes for $M|e|$ to grow from 0 to $\max \{|x|, \eta\}$. For any $q \in \mathbb{R}^{n_{q}} \backslash \mathcal{A}$, we define $\psi(q)=\frac{|e|}{\max \{|x|, \eta\}}$ when $|e| \leq M^{-1} \max \{|x|, \eta\}$ and $\psi(q)=M^{-1}$ otherwise. We note that $\psi$ is indeed locally Lipschitz on an open set containing $\mathcal{S}(\bar{\Delta})=R_{\leq}^{-1}(\bar{\Delta}) \backslash \mathcal{A}$. Let $q \in \mathcal{S}(\bar{\Delta}) \cap D$ and $G(q) \in \mathcal{S}(\bar{\Delta})$. It holds that $G(q)=$ $(x, 0, W(e))$, hence $\psi(G(q))=0$ : item (ii-a) of Proposition 1 holds with $a=0$. In view of the above discussions, item (ii-b) of Proposition 1 is satisfied with $b=M^{-1}$. For almost all $q \in \mathcal{S}(\bar{\Delta}),\langle\nabla|x|, f(x, e)\rangle \leq L_{1}(|x|+|e|) \leq$ $L_{1}(\max \{|x|, \eta\}+|e|)$ for some $L_{1} \geq 0$ in view of Assumption 2 and $\langle\nabla \eta,-\delta(\eta)\rangle \leq L_{4} \eta \leq L_{4} \max \{|x|, \eta\}$ for some $L_{4} \geq 0$ as $\delta$ is locally Lipschitz. Thus, for almost all $q \in \mathcal{S}(\bar{\Delta})$, $\max \{\langle\nabla|x|, f(x, e)\rangle,\langle\nabla \eta,-\delta(\eta)\rangle\} \leq N(\max \{|x|, \eta\}+|e|)$ for some $N \geq 0$. Consequently, for almost all $q \in \mathcal{S}(\bar{\Delta})$,

$$
\langle\nabla \psi(q), F(q)\rangle \leq \frac{|g(x, e)| \max \{|x|, \eta\}+N|e|(\max \{|x|, \eta\}+|e|)}{\max \{|x|, \eta\}^{2}},
$$

since $|g(x, e)| \leq L_{3}(|x|+|e|) \leq L_{3}(\max \{|x|, \eta\}+|e|)$ for some $L_{3} \geq 0$ according to Assumption 2, $\langle\nabla \psi(q), F(q)\rangle \leq$ $\frac{L_{3}(\max \{|x|, \eta\}+|e|) \max \{|x|, \eta\}+N|e|(\max \{|x|, \eta\}+|e|)}{L^{2}}$

$\max \{|x|, \eta\}^{2}$
$L_{3} \max \{|x|, \eta\}^{2}+L_{3}|e| \max \{|x|, \eta\}+N|e| \max \{|x|, \eta\}+N|e|^{2}$

$\max \{|x| \eta \eta\}^{2}$ $L_{3}+\left(L_{3}+N\right) \psi(q)+N \psi(q)^{2}$. Hence item (ii-c) of Proposition 1 holds with $\lambda(s)=L_{3}+\left(L_{3}+N\right) s+N s^{2}$ for any $s \geq 0$. By applying Proposition 1, item (iv) of Theorem 1 is verified and we derive that $\mathcal{A}$ is UGpAS. We note that $\mathcal{A}$ is compact and that $G(D) \subset C \cup D$. Let $q \in C \backslash D$, if $q$ belongs to the interior of $C, T_{C}(q)=\mathbb{R}^{n_{x}+n_{e}+1}$ and $F(q) \in T_{C}(q)$. Otherwise $q$ is such that $\eta=0$, in this case $T_{C}(q)=\mathbb{R}^{n_{x}+n_{e}} \times \mathbb{R}_{>0}$ and $F(q)=(f(x, e), g(x, e), 0) \in T_{C}(q)$. According to Theorem $1, \mathcal{A}$ is UGAS.

Proof of Theorem 3. We consider the locally Lipschitz function, for all $q \in \mathbb{R}^{n_{q}}$,

$$
R(q)=V(x)+\eta W(e)^{2},
$$

which satisfies item (i) of Theorem 1 with $\bar{\alpha}_{R}(s)=\bar{\alpha}_{V}(s)+$ $\bar{c} \bar{\alpha}_{W}(s)^{2}$ and $\underline{\alpha}_{R}(s)=\min \left\{\underline{\alpha}_{V}\left(\frac{s}{2}\right), \underline{c}_{W}\left(\frac{s}{2}\right)^{2}\right\}$ for $s \geq 0$ using Assumption 3 and the fact that $\eta \in[\underline{c}, \bar{c}]$ on $C \cup D \cup G(D)$. For almost all $q \in C,\langle\nabla R(q), F(q)\rangle=\langle\nabla V(x), f(x, e)\rangle+$ $\eta\left\langle\nabla W(e)^{2}, g(x, e)\right\rangle+\left(-2 \eta L(x, e)-\eta^{2}-G(x, e)\right) W(e)^{2}$. From Assumption 3 and in view of (23)-(24) in [30], for all $q \in C$,

$$
\begin{aligned}
R^{\circ}(q ; F(q)) \leq & -\varrho(|x|)-\varrho(|e|)-H^{2}(x)+G(x, e) W(e)^{2} \\
& +\left(-2 \eta L(x, e)-\eta^{2}-G(x, e)\right) W(e)^{2} \\
& +2 \eta W(e)(L(x, e) W(e)+H(x)) \\
= & -\varrho(|x|)-\varrho(|e|)-H^{2}(x)-\eta^{2} W(e)^{2} \\
& +2 \eta H(x) W(e) .
\end{aligned}
$$

Therefore, for all $q \in C$,

$$
\begin{aligned}
R^{\circ}(q ; F(q)) \leq & -\varrho(|x|)-\varrho(|e|)-H^{2}(x)-\eta^{2} W(e)^{2} \\
& +2 \eta H(x) W(e) .
\end{aligned}
$$

Using the fact that $2 \eta W(e) H(x) \leq \eta^{2} W(e)+H^{2}(x)$, we obtain $R^{\circ}(q ; F(q)) \leq-\varrho(|x|)-\varrho(|e|) \leq-\rho(|(x, e)|)$ for some class- $\mathcal{K}$ function $\rho$. Consequently, item (ii) of Theorem 1 is satisfied with $\alpha_{R}=\rho \circ \bar{\alpha}_{R}^{-1}$. Let $q \in D, R(G(q))=$ $V(x) \leq R(q)$ : item (iii) of Theorem 1 is ensured. We apply 
Proposition 1 to show that the solutions to (25), (26) have a uniform semiglobal dwell-time. Let $\Delta \geq 0$ and define $\psi(q)=$ $\frac{1}{\eta}$ which is well-defined on an open set containing $R_{\leq}^{-1}(\Delta)$ (since $\eta \in[\underline{c}, \bar{c}]$ on $C \cup D \cup G(D)$ ). Let $q \in R_{<}^{-1}(\Delta) \cap D$, $\psi(G(q))=\bar{c}^{-1}$ : item (ii-a) of Proposition 1 holds with $a=$ $\bar{c}^{-1}$. In view of the definition of the jump set in (26), for any $q \in R_{<}^{-1}(\Delta), \psi(q)<\underline{c}^{-1}$ implies $q \in C \backslash D$ : item (iib) of Proposition 1 holds with $b=\underline{c}^{-1}$. Noting that $\psi$ is continuously differentiable on $R_{\leq}^{-1}(\Delta)$, let $q \in R_{\leq}^{-1}(\Delta)$

$$
\langle\nabla \psi(q), F(q)\rangle=-\frac{\left\langle\nabla \eta,-2 \eta L(x, e)-\eta^{2}-G(x, e)\right\rangle}{\eta^{2}} .
$$

Since $q \in R_{\leq}^{-1}(\Delta), R_{<}^{-1}(\Delta)$ is compact and $L, G$ are continuous, there exists $M \geq 0$ such that $\max \{L(x, e), G(x, e)\} \leq$ $M$, thus

$$
\langle\nabla \psi(q), F(q)\rangle \leq \frac{2 \eta M+\eta^{2}+M}{\eta^{2}}
$$

and item (ii-c) of Proposition 1 holds with $\lambda(s)=2 M s+1+M s^{2}$ for any $s \geq 0$. Proposition 1 guarantees that the solutions to (25), (26) have a uniform semiglobal dwell-time (since $\mathcal{A}$ is compact). Noting that $\mathcal{A}$ is compact, $G(D) \subset C \cup D$, we only need to prove that $F(q) \in T_{C}(q)$ for any $q \in C \backslash D$ to conclude the proof using Theorem 1. Let $q \in C \backslash D$, when $\eta \in(\underline{c}, \bar{c})$ the result holds as $T_{C}(q)=\mathbb{R}^{n_{x}+n_{e}+1}$. Suppose $\eta=\bar{c}, T_{C}(q)=\mathbb{R}^{n_{x}+n_{e}} \times(-\infty, 0]$. Since $F(q)=\left(f(x, e), g(x, e),-2 \eta L(x, e)-\eta^{2}-G(x, e)\right)$ and $L$ and $G$ are non-negative, $F(q) \in T_{C}(q)$. As a consequence, $\mathcal{A}$ is UGAS in view of Theorem 1 .

Proof of Theorem 5. We consider the following locally Lipschitz function $R(q):=R_{1}(x)+R_{2}(e)$ for all $q \in \mathbb{R}^{n_{q}}$, where

$$
\begin{aligned}
& R_{1}(x)=\max \left\{V(x)-\alpha^{-1}(2 \rho), 0\right\} \\
& R_{2}(e)=\max \{\gamma(|e|)-\rho, 0\} .
\end{aligned}
$$

We note that $R$ only becomes equal to zero on the compact set $\mathcal{A}=\left\{(x, e): V(x) \leq \alpha^{-1}(2 \rho)\right.$ and $\left.\gamma(|e|) \leq \rho\right\}$, is continuous and tends to $\infty$ only as $|q|_{\mathcal{A}} \rightarrow \infty$. Hence item (i) of Theorem 1 holds by applying Lemma 3. Let $q \in C$. When $V(x)-\alpha^{-1}(2 \rho)>0$, it holds that, in view of (15) and Lemma 1,

$$
\begin{aligned}
R_{1}^{\circ}(x ; f(x, e)) & =\langle\nabla V(x), f(x, e)\rangle \leq-\alpha(V(x))+\gamma(|e|) \\
\leq & -\alpha(V(x))+\rho
\end{aligned}
$$

since on the set $C$ we have $R(q)=R_{1}(x)$, we derive that

$$
\begin{aligned}
R_{1}^{\circ}(x ; f(x, e)) & \leq-\alpha\left(R_{1}(x)+\alpha^{-1}(2 \rho)\right)+\rho \\
& =-\alpha\left(R(q)+\alpha^{-1}(2 \rho)\right)+\rho .
\end{aligned}
$$

We note that $\frac{1}{2} \alpha(R(q))+\rho=\frac{1}{2} \alpha(R(q))+$ $\frac{1}{2} \alpha \circ \alpha^{-1}(2 \rho) \stackrel{\leq}{\leq} \alpha\left(R(q)+\alpha^{-1}(2 \rho)\right)$ since $\alpha$ is strictly increasing as a class- $\mathcal{K}_{\infty}$ function. Therefore $R_{1}^{\circ}(x ; f(x, e)) \leq-\frac{1}{2} \alpha(R(q))-\rho+\rho=-\frac{1}{2} \alpha(R(q))$. In view of Lemma 1 and above, when $V(x)-\alpha^{-1}(2 \rho) \leq 0$, we have that $R_{1}^{\circ}(x ; f(x, e)) \leq 0=-\frac{1}{2} \alpha(R(q))$ as $q \in \mathcal{A}$ and $R$ is equal to zero on $\mathcal{A}$. We now consider $R_{2}$ still for $q \in C$. Note that we cannot have $\gamma(|e|)-\rho>0$ as $q \in C$. When $\gamma(|e|)-\rho<0, R_{2}^{\circ}(e ; g(x, e))=0$. Consider the case where $\gamma(|e|)-\rho=0$, i.e. $q \in C \cap D$ and assume $F(q) \in T_{C}(q)$. According to Lemma 4, $0=\langle\nabla \rho, F(q)\rangle \geq\langle\nabla \gamma(|e|), g(x, e)\rangle$, hence $R_{2}^{\circ}(e ; g(x, e))=\max \{0,\langle\nabla \gamma(|e|), g(x, e)\rangle\} \leq 0$. We have proved that item (ii) of Theorem 1 is verified with $\alpha_{R}=\frac{1}{2} \alpha$. Let $q \in D, R(G(q))=R_{1}(x)+0 \leq R(q)$ : item (iii) of Theorem 1 holds. We now prove that the solutions to (31), (32) have a uniform semiglobal dwell-time. Define $\psi(q)=\gamma(|e|)$ for $q \in \mathbb{R}^{n_{q}}$, which is locally Lipschitz on an open set containing $R_{<}^{-1}(\Delta)$ as desired. Let $\Delta \geq 0$ and $q \in R_{\leq}^{-1}(\Delta) \cap D, \psi(G(q))=0$ : item (ii-a) of Proposition 1 holds with $a=0$. Let $q \in R_{\leq}^{-1}(\Delta), \psi(q)<\rho$ implies that $q \in C \backslash D$ : item (ii-b) of Proposition 1 is verified with $b=\rho$. Since the function $\psi$ is continuously differentiable, there exists $M \geq 0$ such that for almost all $q \in R_{<}^{-1}(\Delta)$, $\langle\nabla \gamma(|e|), g(x, e)\rangle \leq M$. Hence item (ii-c) of Proposition 1 is verified with $\lambda(s)=M$ for $s \geq 0$. We deduce that the solutions to (31), (32) have a uniform semiglobal dwell-time by applying Proposition 1 (as $\mathcal{A}$ is compact). According to Theorem $1, \mathcal{A}$ is UGpAS. Note that $\mathcal{A}$ is compact and $G(D) \subset C \cup D$. Let $q \in C \backslash D$, i.e. $\gamma(|e|)<\rho$, then $F(q) \in T_{C}(q)=\mathbb{R}^{n_{x}+n_{e}}$. Consequently, $\mathcal{A}$ is UGAS in view of Theorem 1 .

Proof of Theorem 6. We consider $R(q)=\max \{\eta, V(x)\}$ which is locally Lipschitz on $\mathbb{R}^{n_{q}}$. Item (i) of Theorem 1 holds with $\bar{\alpha}_{R}(s)=\max \left\{s, \bar{\alpha}_{V}(s)\right\}$ and $\underline{\alpha}_{R}(s)=\frac{1}{2} \min \{s / 2$, $\left.\underline{\alpha}_{V}(s / 2)\right\}$ for $s \geq 0$. Let $q \in C$ and $F(q) \in T_{C}(q)$. When $\eta>V(x), R^{\circ}(q ; F(q))=-\delta(\eta)$ according to Lemma 1. Hence $R^{\circ}(q ; F(q))=-\delta(R(q))$. When $\eta=V(x)$, $R^{\circ}(q ; F(q))=\max \{\langle\nabla V(x), f(x, e)\rangle,-\delta(\eta)\}$ according to Lemma 1. We apply Lemma 4 with $V(x)=\eta$ and $W(x)=V(x)$ to derive that $\langle\nabla V(x), f(x, e)\rangle \leq-\delta(\eta)$, therefore $R^{\circ}(q ; F(q))=-\delta(\eta)=-\delta(R(q))$. Consequently, item (ii) of Theorem 1 is verified with $\alpha_{R}=\delta$. Let $q \in D$, $R(G(q))=V(x) \leq R(q)$ : item (iii) of Theorem 1 holds. From the fact that $\delta \leq \sigma \circ \alpha$, the next jump instant cannot occur before $W(e)$ grows from 0 to $\sigma \circ \alpha(V(x))$, which corresponds to the event-triggering condition studied in Section V-C. We know from Theorem 4 that this time is lower bounded by a uniform semiglobal constant as long we are not in $\tilde{\mathcal{A}}:=\{q:(x, e)=0\}$, since Assumptions 1-2 are verified. On the other hand, if a solution lies in $\tilde{\mathcal{A}}$ with $\eta \neq 0$, no jump will occur as $V(x)$ will never become equal to $\eta$. As a consequence, the solutions to (35), (36) have a uniform semiglobal dwell-time outside the origin, and hence outside $\mathcal{A}$. We conclude the proof by applying Theorem 1 . $\square$

\section{REFERENCES}

[1] K.E. Arzén. A simple event-based PID controller. In Proceedings of the 14th IFAC World Congress, Beijing, China, volume 18, pages 423-428, 1999.

[2] K.J. Aström and B.M. Bernhardsson. Comparison of periodic and event based sampling for first-order stochastic systems. In Proceedings of the 14th IFAC World congress, Beijing, China, volume 11, pages 301-306, 1999.

[3] K.J. Aström and B.M. Bernhardsson. Comparison of Riemann and Lebesgue sampling for first order stochastic systems. In IEEE Conference on Decision and Control, Las Vegas, U.S.A., pages 2011-2016, 2002. 
[4] F. H. Clarke. Optimization and nonsmooth analysis. Classics in Applied Mathematics vol. 5, SIAM, Philadelphia, U.S.A., 1990.

[5] M.C.F. Donkers and W.P.M.H. Heemels. Output-based event-triggered control with guaranteed $\mathcal{L}_{\infty}$-gain and improved and decentralized eventtriggering. IEEE Transactions on Automatic Control, 57(6):1362-1376, 2012.

[6] F. Forni, S. Galeani, D. Nešić, and L. Zaccarian. Lazy sensors for the scheduling of measurement samples transmission in linear closed loops over networks. In IEEE Conf. on Decision and Control, Atlanta, U.S.A., pages 6469-6474, 2010.

[7] R. Goebel, R.G. Sanfelice, and A.R. Teel. Invariance principles for switching systems via hybrid systems techniques. Systems \& Control Letters, 57:980-986, 2008.

[8] R. Goebel, R.G. Sanfelice, and A.R. Teel. Hybrid dynamical systems. Princeton, U.S.A., 2012.

[9] L. Grüne. Asymptotic behavior of dynamical and control systems under pertubation and discretization. Number 1783. Springer, 2002.

[10] W.P.M.H. Heemels, M.C.F. Donkers, and A.R. Teel. Periodic eventtriggered control for linear systems. IEEE Transactions Automatic Control, 58(4):847-861, 2013.

[11] W.P.M.H. Heemels, J.H. Sandee, and P.P.J. van den Bosch. Analysis of event-driven controllers for linear systems. International Journal of Control, 81(4):571-590, 2009.

[12] H.K. Khalil. Nonlinear systems. Prentice-Hall, Englewood Cliffs, New Jersey, U.S.A., 3rd edition, 2002.

[13] E. Kofman and J.H. Braslavsky. Level crossing sampling in feedback stabilization under data-rate constraints. In IEEE Conference on Decision and Control, pages 4423-4428, 2006.

[14] M. Krstić and P.V. Kokotović. Lean backstepping design for a jet engine compressor model. In Proceedings of the 4th IEEE Conference on Control Applications, pages 1047-1052, 1995.

[15] J. Lunze and D. Lehmann. A state-feedback approach to event-based control. Automatica, 46:211-215, 2010.

[16] M. Miskowicz. Send-on-delta concept: An event-based data reporting strategy. Sensors, 6(1):49-63, 2006.

[17] D. Nešić and A.R. Teel. Input-output stability properties of networked control systems. IEEE Trans. on Aut. Control, 49:1650-1667, 2004.

[18] D. Nešić and A.R. Teel. A Lyapunov-based small-gain theorem for hybrid ISS systems. In IEEE Conference on Decision and Control, Cancun, Mexico, pages 3380-3385, 2008.

[19] D. Nešić, A.R. Teel, and D. Carnevale. Explicit computation of the sampling period in emulation of controllers for nonlinear sampled-data systems. IEEE Transactions on Automatic Control, 54(3):619-624, 2009.

[20] G. Otanez, J.R. Moyne, and D.M. Tilbury. Using deadbands to reduce communication in networked control systems. In American Control Conference, Anchorage, U.S.A., pages 3015-3020, 2002.

[21] C. De Persis and R. Postoyan. A Lyapunov redesign of coordination algorithms for cyberphysical systems. In arXiv 1404.0576, 2014.

[22] R. Postoyan, A. Anta, W.P.M.H. Heeemels, P. Tabuada, and D. Nešić. Periodic event-triggered control for nonlinear systems. In IEEE Conference on Decision and Control, Florence, Italy, 2013.

[23] R. Postoyan, A. Anta, D. Nešić, and P. Tabuada. A unifying Lyapunovbased framework for the event-triggered control of nonlinear systems. In IEEE Conference on Decision and Control and European Control Conference, Orlando, U.S.A., pages 2559-2564, 2011.

[24] R. Postoyan, P. Tabuada, D. Nešić, and A. Anta. Event-triggered and self-triggered stabilization of distributed networked control systems. In IEEE Conf. on Decision and Control and European Control Conf., Orlando, U.S.A., pages 2565-2570, 2011.

[25] S. Prajna, A. Papachristodoulou, P. Seiler, and P.A. Parrilo. Sostools: sum of squares optimization toolbox for matlab. URL: http://www.cds.caltech.edu/sostools, 2004.

[26] A. Seuret, C. Prieur, and N. Marchand. Stability of nonlinear systems by means of event-triggered sampling algorithms. IMA Journal of Mathematical Control and Information, 31(3):415-433, 2013.

[27] E.D. Sontag and Y. Wang. On characterizations of the input-to-state stability property. Systems \& Control Letters, 24(5):351-359, 1995.

[28] P. Tabuada. Event-triggered real-time scheduling of stabilizing control tasks. IEEE Transactions on Automatic Control, 52(9):1680-1685, 2007.

[29] P. Tallapragada and N. Chopra. On event triggered tracking for nonlinear systems. IEEE Transactions on Automatic Control, 58(9):2343-2348, 2013.

[30] A.R. Teel and L. Praly. On assigning the derivative of a disturbance attenuation control Lyapunov function. Mathematics of Control, Signals and Systems, 13(2):95-124, 2000.
[31] M. Velasco, P., Martí, and E. Bini. On Lyapunov sampling for eventdriven controllers. In IEEE Conference on Decision and Control held jointly with the Chinese Control Conference, Shangaï, China, pages 6238-6243, 2009.

[32] G.C. Walsh, O. Beldiman, and L.G. Bushnell. Asymptotic behavior of nonlinear networked control systems. IEEE Transactions on Automatic Control, 46:1093-1097, 2001.

[33] X. Wang and M.D. Lemmon. Event design in event-triggered feedback control systems. In IEEE Conference on Decision and Control, Cancun, Mexico, pages 2105-2110, 2008.

[34] X. Wang and M.D. Lemmon. On event design in event-triggered feedback systems. Automatica, 47(10):2319-2322, 2011.

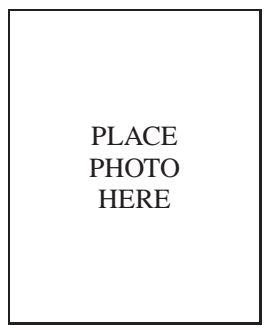

Romain Postoyan was born in Paris, France, in 1982. He received the M.Sc. degree in Electrical and Control Engineering from ENSEEIHT (France) in 2005. He obtained the M.Sc. by Research in Control Theory \& Application from Coventry University (United Kingdom) in 2006 and the Ph.D. in Control Theory from Université Paris-Sud (France) in 2009. In 2010, he was a research assistant at the University of Melbourne (Australia). Since 2011, he is a CNRS researcher at the Centre de Recherche en Automatique de Nancy (France).

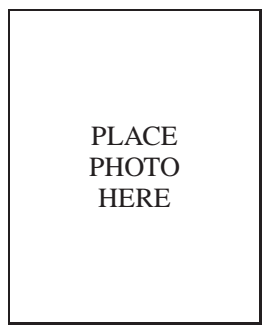

Paulo Tabuada was born in Lisbon, Portugal, one year after the Carnation Revolution. He received his 'Licenciatura' degree in Aerospace Engineering from Instituto Superior Tecnico, Lisbon, Portugal in 1998 and his Ph.D. degree in Electrical and Computer Engineering in 2002 from the Institute for Systems and Robotics, a private research institute associated with Instituto Superior Tecnico. Between January 2002 and July 2003 he was a postdoctoral researcher at the University of Pennsylvania. After spending three years at the University of Notre Dame, as an Assistant Professor, he joined the Electrical Engineering Department at the University of California, Los Angeles, where he established and directs the Cyber-Physical Systems Laboratory.

Paulo Tabuada's contributions to cyber-physical systems have been recognized by multiple awards including the NSF CAREER award in 2005, the Donald P. Eckman award in 2009 and the George S. Axelby award in 2011. In 2009 he co-chaired the International Conference Hybrid Systems: Computation and Control (HSCC'09), in 2012 he was program co-chair for the 3rd IFAC Workshop on Distributed Estimation and Control in Networked Systems (NecSys'12), and in 2015 he was the program co-chair for the IFAC Conference on Analysis and Design of Hybrid Systems. He also served on the editorial board of the IEEE Embedded Systems Letters and the IEEE Transactions on Automatic Control. His latest book, on verification and control of hybrid systems, was published by Springer in 2009 . 


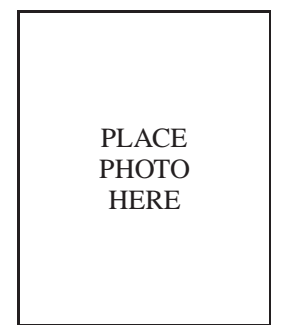

Dragan Nešić is a Professor in the Department of Electrical and Electronic Engineering (DEEE) at The University of Melbourne, Australia. He received his BE degree in Mechanical Engineering from The University of Belgrade, Yugoslavia in 1990, and his Ph.D. degree from Systems Engineering, RSISE, Australian National University, Canberra, Australia in 1997. Since February 1999 he has been with The University of Melbourne. His research interests include networked control systems, discrete-time, sampled-data and continuous-time nonlinear control systems, input-to-state stability, extremum seeking control, applications of symbolic computation in control theory, hybrid control systems, and so on. He was awarded a Humboldt Research Fellowship (2003) by the Alexander von Humboldt Foundation, an Australian Professorial Fellowship (2004-2009) and Future Fellowship (2010-2014) by the Australian Research Council. He is a Fellow of IEEE and a Fellow of IEAust. He is currently a Distinguished Lecturer of CSS, IEEE (2008-). He served as an Associate Editor for the journals Automatica, IEEE Transactions on Automatic Control, Systems and Control Letters and European Journal of Control.

\begin{tabular}{|c|} 
\\
\\
PLACE \\
PHOTO \\
HERE \\
\end{tabular}

Adolfo Anta received the 'Licenciatura' degree from ICAI Engineering School, Madrid, Spain, in 2002, and the M.Sc. and Ph.D. degrees from the University of California, Los Angeles, CA, USA, in 2007 and 2010, respectively. From 2002 to 2005, he was a Design Engineer with EADS and, from 2010 to 2012, he was a Postdoctoral Researcher with the Technical University of Berlin and the Max Planck Institute, Germany. He is currently with General Electric Global Research Europe. His current research interests include robust control, networked control systems, and power systems.

Dr. Anta received the Fulbright Scholarship in 2005, the Alexander von Humboldt Fellowship in 2011, was a Finalist for the Student Best Paper Award at the IEEE Conference on Decision and Control in 2008, received the 2010 EMSOFT Best Paper Award and the IEEE CSS George S. Axelby Award in 2011. 